\title{
Heavy Neutrinos and Lepton Flavour Violation in Left-Right Symmetric Models at the LHC
}

\author{
S. P. Das, ${ }^{1, *}$ F. F. Deppisch, ${ }^{2, \oplus}$ O. Kittel, ${ }^{3, \text { f }}$ and J. W. F. Valle ${ }^{4, \text { S }}$ \\ ${ }^{1}$ Department of Physics, Visva-Bharati, Santiniketan - 731 235, India \\ ${ }^{2}$ Department of Physics and Astronomy, University College London, \\ London WC1E 6BT, United Kingdom \\ ${ }^{3}$ Departamento de Física Teórica y del Cosmos and CAFPE, \\ Universidad de Granada, E-18071 Granada, Spain \\ ${ }^{4}$ AHEP Group, Institut de Física Corpuscular - C.S.I.C./Universitat de València \\ Edificio Institutos de Paterna, Apt 22085, E-46071 Valencia, Spain

\begin{abstract}
We discuss lepton flavour violating processes induced in the production and decay of heavy right-handed neutrinos at the LHC. Such particles appear in left-right symmetrical extensions of the Standard Model as the messengers of neutrino mass generation, and can have masses at the $\mathrm{TeV}$ scale. We determine the expected sensitivity on the right-handed neutrino mixing matrix, as well as on the righthanded gauge boson and heavy neutrino masses. By comparing the sensitivity of the LHC with that of searches for low energy LFV processes, we identify favourable areas of the parameter space to explore the complementarity between LFV at low and high energies.
\end{abstract}

\footnotetext{
*Electronic address: spdas@ific.uv.es

$\dagger$ Electronic address: f.deppisch@ucl.ac.uk

¥Electronic address: kittel@th.physik.uni-bonn.de

${ }^{\S}$ Electronic address: valle@ific.uv.es
} 


\section{INTRODUCTION}

The discovery of neutrino oscillations [1-3] shows that neutrinos are massive [4] and that lepton flavour is violated in neutrino propagation. It is natural to expect that the violation of this conservation law should show up in other contexts, such as rare lepton flavour violating (LFV) decays of muons and taus, e.g. $\mu^{-} \rightarrow e^{-} \gamma$, and possibly also at the high energies accessible at the Large Hadron Collider (LHC). In addition to proving that flavour is violated in the leptonic sector, oscillation experiments have convincingly shown that at least two of the three active neutrinos have a finite mass. However, despite this success, oscillation experiments are unable to determine the absolute magnitude of neutrino masses. Altogether there are three complementary approaches to probe the absolute scale of neutrino mass. Upper limits on the effective electron neutrino mass of $\sim 2 \mathrm{eV}$ can be set from the analysis of tritium beta-decay experiments [5, 6]. Astronomical observations combined with cosmological considerations also allow an upper bound to be set on the sum of the three neutrino masses of the order of $0.7 \mathrm{eV}$, under some assumptions [7]. The observation of neutrinoless double beta decay $(0 \nu \beta \beta)$ would signal the Majorana nature of neutrinos and the violation of total lepton number [8, 9]. Corresponding searches provide an upper bound on the $0 \nu \beta \beta$ effective Majorana neutrino mass parameter $m_{\beta \beta} \leq 300-600 \mathrm{meV}$ [10]. Again, it is to be expected that, if $0 \nu \beta \beta$ is observed, the violation of total lepton number should also, at some level, take place at high energy accelerators like the LHC.

Several mechanisms of neutrino mass generation have been suggested in the literature, the most prominent example being the seesaw mechanism in which heavy right-handed Majorana neutrinos act as "messengers" by generating light Majorana masses for the observed active neutrinos through their mixing with the left-handed neutrinos. The Majorana character of the active neutrinos can then be connected to a breaking of lepton number symmetry at a scale possibly associated with unification [11 17] and might also be responsible for the baryon asymmetry of the Universe through the leptogenesis mechanism [18].

Despite its attractiveness, the default type-I seesaw mechanism has important phenomenological shortcomings: In the standard regime, the right-handed neutrinos have masses close to the unification scale and can therefore not be directly produced. In addition, the right-handed neutrinos are gauge singlets. This means that even if the masses are low enough for them to be produced, the heavy neutrinos only couple with Yukawa strength, tightly constrained by the smallness of neutrino masses ${ }^{1}$. This implies that the simplest seesaw schemes are difficult to test at the LHC [22].

A widely studied alternative of the standard Seesaw scheme with gauge singlet heavy neutrinos is the left-right symmetrical model (LRSM) which extends the electroweak Standard Model gauge symmetry $\mathrm{SU}(2)_{L} \otimes \mathrm{U}(1)$ to the $\mathrm{SU}(2)_{L} \otimes \mathrm{SU}(2)_{R} \otimes \mathrm{U}(1)_{B-L}$ group [2326]. Here, right-handed neutrinos are necessary to realize the extended gauge symmetry and come as part of an $\mathrm{SU}(2)_{R}$ doublet, coupling to the heavy gauge bosons. As a result, heavy neutrinos can be produced with gauge coupling strength, with promising discovery prospects, given the relatively weak direct experimental bounds on the masses of the extra gauge bosons.

In this paper we address the prospects of probing the mass matrix of the right-handed neutrinos. With the existing information on the light neutrino sector and low energy lepton

\footnotetext{
${ }^{1}$ The mixing of the heavy neutrino singlets is also constrained by precision data such as the rates for lepton flavour violating processes 19 21].
} 


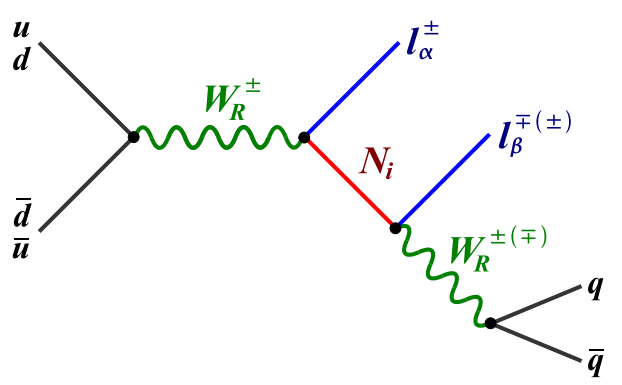

(a)

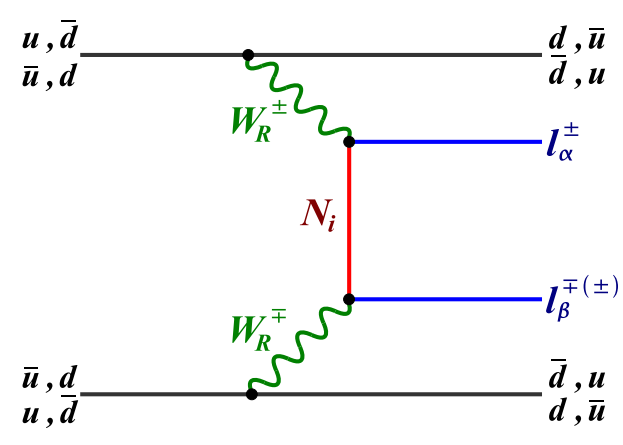

(b)

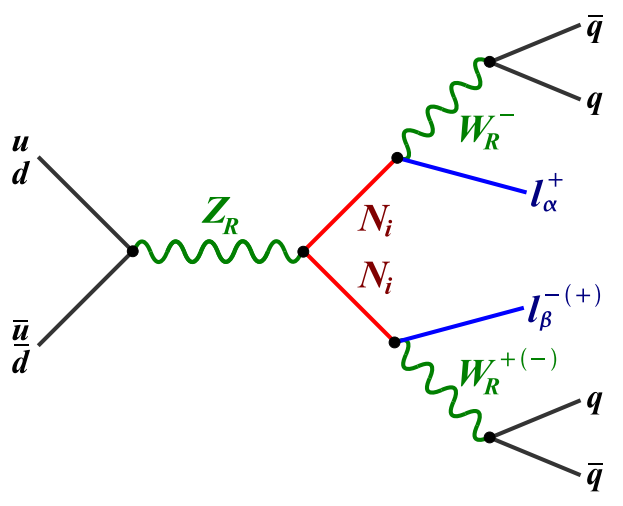

(c)

FIG. 1: Different production processes of heavy right-handed neutrinos with dilepton signatures at hadron colliders.

flavour violation, we re-derive the expected LHC sensitivities on the right-handed gauge boson and heavy neutrino masses. Among the possible production processes shown in Figure 1, we will focus on the resonant production of a right-handed $W_{R}$ boson displayed in Figure 1(a), In addition, we determine the sensitivity of lepton flavour violating processes at the LHC to measure the right-handed neutrino masses and their mixing, and compare results with low energy LFV searches. The focus in this paper is on lepton flavour violating effects rather than lepton number violation, and we will take into account both opposite-sign and same-sign lepton events of the process in Figure 1(a) at the LHC. Nevertheless, we will briefly comment on dedicated searches of lepton number violation using same-sign event signatures and the interplay with $0 \nu \beta \beta$.

This paper is organized as follows. In Section $\amalg$ we introduce the minimal LR model, and discuss the structure of its charged current couplings. Section III covers the main observables which give current limits on the LR model used. The dilepton signals arising from heavy neutrino production are discussed in Section IV] Finally, we present our conclusions in Section $\nabla$. 


\section{LEFT-RIGHT SYMMETRY}

A striking feature of neutrino oscillation data is that the leptonic mixing angles follow a pattern substantially different from that which characterizes quarks [27]. Within the seesaw mechanism, as implemented in left-right symmetrical models, neutrino masses are generated in a different way from charged fermion masses, as a result of the violation of lepton number. Hence, in principle, one may reconcile the large solar and atmospheric angles indicated by neutrino oscillation data with the small Kobayashi-Maskawa angles. Many specific models based on flavour symmetries have been suggested for this [28]. In what follows we will simply assume a generic lepton flavour structure with the right-handed neutrinos and the charged gauge bosons of the left-right model in the range of a few $\mathrm{TeV}$.

\section{A. Minimal Left-Right Symmetrical Model}

In the minimal $\mathrm{SU}(2)_{L} \otimes \mathrm{SU}(2)_{R} \otimes \mathrm{U}(1)_{B-L}$ model with manifest left-right symmetry, a generation of quarks and leptons is assigned to the multiplets [29]

$$
Q_{L, R}=\left(\begin{array}{l}
u \\
d
\end{array}\right)_{L, R}, \quad \psi_{L, R}=\left(\begin{array}{l}
\nu \\
\ell
\end{array}\right)_{L, R},
$$

with the quantum numbers under $\mathrm{SU}(2)_{L} \otimes \mathrm{SU}(2)_{R} \otimes \mathrm{U}(1)_{B-L}$

$$
\begin{array}{ll}
Q_{L}:\left(\frac{1}{2}, 0, \frac{1}{3}\right), & \psi_{L}:\left(\frac{1}{2}, 0,-1\right), \\
Q_{R}:\left(0, \frac{1}{2}, \frac{1}{3}\right), & \psi_{R}:\left(0, \frac{1}{2},-1\right) .
\end{array}
$$

The Higgs sector contains a bidoublet

$$
\phi=\left(\begin{array}{cc}
\phi_{1}^{0} & \phi_{1}^{+} \\
\phi_{2}^{-} & \phi_{2}^{0}
\end{array}\right):\left(\frac{1}{2}, \frac{1}{2}^{*}, 0\right),
$$

and two scalar triplets $\Delta_{L, R}$ needed to break the right-handed symmetry,

$$
\Delta_{L, R}=\left(\begin{array}{cc}
\frac{\Delta_{L, R}^{+}}{\sqrt{2}} & \Delta_{L, R}^{++} \\
\Delta_{L, R}^{0} & \frac{-\Delta_{L, R}^{+}}{\sqrt{2}}
\end{array}\right),
$$

with the quantum numbers $\Delta_{L}:(1,0,2)$ and $\Delta_{R}:(0,1,2)$, respectively. This choice is by no means unique and there are other multiplet choices that ensure a satisfactory symmetry breaking pattern (we will comment on possible alternatives in the conclusion). For our analysis we adopt, for concreteness, this more standard choice. The symmetry breaking is triggered by the following vacuum expectation values (VEVs)

$$
\langle\phi\rangle=\left(\begin{array}{cc}
\frac{k_{1}}{\sqrt{2}} & 0 \\
0 & \frac{k_{2}}{\sqrt{2}}
\end{array}\right), \quad\left\langle\Delta_{L, R}\right\rangle=\left(\begin{array}{cc}
0 & 0 \\
\frac{v_{L, R}}{\sqrt{2}} & 0
\end{array}\right),
$$

where $v_{R}$ of the right triplet breaks $\mathrm{SU}(2)_{R} \otimes \mathrm{U}(1)_{B-L}$ to $\mathrm{U}(1)_{Y}$ and provides masses for the new heavy particles. As these new fields have not been observed and due to the strong 
limits on right-handed currents, $v_{R}$ should be sufficiently large. On the other hand, the VEV $v_{L}$ of the left triplet contributes to the $\rho$ parameter, and is therefore experimentally constrained to values $\lesssim 5 \mathrm{GeV}$ (see for example [30], and references therein). Finally, the bidoublet VEVs $k_{1}$ and $k_{2}$ break the Standard Model symmetry and are of the order of the electroweak scale. Consequently, the VEVs follow the hierarchy $\left|v_{L}\right| \ll\left|k_{i}\right| \ll\left|v_{R}\right|$. In the following we assume that all VEVs are real and we neglect the possibility of $C P$ violating phases.

The leptonic Yukawa Lagrangian under $\mathrm{SU}(2)_{L} \otimes \mathrm{SU}(2)_{R} \otimes \mathrm{U}(1)_{B-L}$ is given by [29]

$$
-\mathcal{L}=\bar{\psi}_{L} Y_{1} \phi \psi_{R}+\bar{\psi}_{L} Y_{2} \tilde{\phi} \psi_{R}+\psi_{L}^{T}\left(i Y_{M}\right) \Delta_{L} \psi_{L}+\psi_{R}^{T}\left(i Y_{M}\right) \Delta_{R} \psi_{R}+\text { h.c. },
$$

where we suppress gauge invariant field contractions and the summation over the flavour indices of the fermion fields and the $3 \times 3$ Yukawa matrices $Y_{1}, Y_{2}$ and $Y_{M}$.

The Lagrangian (6) leads to the $6 \times 6$ neutrino mass matrix $M_{\nu}$ with Dirac mass terms arising from $Y_{1}$ and $Y_{2}$ as well as Majorana mass terms originating from $Y_{M}$. The mass matrix can be written in block form

$$
M_{\nu}=\left(\begin{array}{cc}
M_{L} & M_{D} \\
M_{D}^{T} & M_{R}
\end{array}\right)
$$

in the basis $\left(\nu_{L}, \nu_{L}^{c}\right)^{T}$. Due to the Pauli principle, $M_{\nu}$ is complex symmetric. The $3 \times 3$ entries of this matrix are given by

$$
M_{L, R}=\sqrt{2} Y_{M} v_{L, R}, \quad M_{D}=\frac{1}{\sqrt{2}}\left(Y_{1} k_{1}+Y_{2} k_{2}\right)
$$

Assuming that all Yukawa couplings (of a given generation) are of similar magnitude, the structure of $M_{\nu}$ follows the hierarchy of the Higgs VEVs, i.e. $M_{L} \ll M_{D} \ll M_{R}$. Equivalently, the Yukawa couplings in (6) lead to the charged lepton mass matrix $M_{\ell}=$ $\frac{1}{\sqrt{2}}\left(Y_{2} k_{1}+Y_{1} k_{2}\right)$. In the following, we will not specify the flavour structure generated by the Yukawa couplings. Instead we will phenomenologically parametrize the charged current couplings between the heavy neutrinos and the charged leptons.

The neutrino mass matrix is diagonalized by the $6 \times 6$ complex orthogonal mixing matrix $U_{\nu}$ as [15]

$$
U_{\nu}^{T} M_{\nu} U_{\nu}=\operatorname{diag}\left(m_{\nu_{1}}, m_{\nu_{2}}, m_{\nu_{3}}, m_{N_{1}}, m_{N_{2}}, m_{N_{3}}\right),
$$

with the light and heavy neutrino masses $m_{\nu_{i}}$ and $m_{N_{i}}$, respectively (the transformation can be chosen such that the mass eigenvalues are positive and appropriately ordered).

Before discussing the generic flavour structure of the charged currents generated in the LRSM, we will briefly summarize the spectrum of heavy particles relevant to our discussion. After both LR and electroweak symmetry breaking and assuming CP invariance, the charged gauge boson eigenstates $W_{L}$ and $W_{R}$ mix as

$$
\left(\begin{array}{l}
W_{1} \\
W_{2}
\end{array}\right)=\left(\begin{array}{cc}
\cos \zeta_{W} & \sin \zeta_{W} \\
-\sin \zeta_{W} & \cos \zeta_{W}
\end{array}\right)\left(\begin{array}{l}
W_{L} \\
W_{R}
\end{array}\right) .
$$

Similarly, the neutral gauge bosons $W_{L}^{3}, W_{R}^{3}$ and $Y$ combining to $Z_{L}$ and $Z_{R}$ mix to form the mass eigenstates

$$
\left(\begin{array}{l}
Z_{1} \\
Z_{2}
\end{array}\right)=\left(\begin{array}{cc}
\cos \zeta_{Z} & \sin \zeta_{Z} \\
-\sin \zeta_{Z} & \cos \zeta_{Z}
\end{array}\right)\left(\begin{array}{l}
Z_{L} \\
Z_{R}
\end{array}\right) .
$$


As outlined in Section 【II, the mixing angles $\zeta_{W}$ and $\zeta_{Z}$ are highly constrained from electroweak precision data. Consequently, the mass eigenstates are essentially given by the gauge eigenstates, and the heavy components have masses of the order of the right-handed symmetry breaking scale, $m_{Z_{R}} \approx m_{W_{R}} \approx v_{R}$.

Generally, the neutrino mass eigenstates are mixtures of the weak eigenstates, forming three light neutrinos $\nu_{i}$ and three heavy neutrinos $N_{i}$, cf. Eq. (9). Neglecting the flavour structure, this leads to the diagonalization in block form

$$
\left(\begin{array}{c}
\nu \\
N
\end{array}\right)=\left(\begin{array}{cc}
\cos \zeta_{\nu} & \sin \zeta_{\nu} \\
-\sin \zeta_{\nu} & \cos \zeta_{\nu}
\end{array}\right)\left(\begin{array}{c}
\nu_{L}+\nu_{L}^{C} \\
\nu_{R}+\nu_{R}^{C}
\end{array}\right)
$$

with the mixing angle $\zeta_{\nu}$ between left- and right-handed neutrinos. For one generation, the charged current weak interactions can then be written in terms of the mass eigenstates as

$$
\begin{aligned}
& J_{W_{1}}^{\mu-}=\frac{g_{L}}{2 \sqrt{2}}\left(\bar{\nu}+\sin \zeta_{\nu} \bar{N}^{c}\right) \gamma^{\mu}\left(1-\gamma_{5}\right) e+\frac{g_{R}}{2 \sqrt{2}} \sin \zeta_{W} \bar{N} \gamma^{\mu}\left(1+\gamma_{5}\right) e \\
& J_{W_{2}}^{\mu-}=-\frac{g_{L}}{2 \sqrt{2}} \sin \zeta_{W} \bar{\nu} \gamma^{\mu}\left(1-\gamma_{5}\right) e+\frac{g_{R}}{2 \sqrt{2}}\left(\bar{N}-\sin \zeta_{\nu} \bar{\nu}^{c}\right) \gamma^{\mu}\left(1+\gamma_{5}\right) e .
\end{aligned}
$$

\section{B. Generic Lepton Flavour Structure}

In order to discuss the flavour structure of the charged current interaction we need to consider the multi-generation case. The effective $3 \times 6$ lepton mixing matrices $\left(U_{L, R}\right)_{\ell i}$, $\ell=e, \mu, \tau, i=1, \ldots, 6$ characterizing the charged (and neutral) current weak interactions of the mass eigenstate neutrinos in any seesaw model have been fully characterized in Ref. [15] and may be written as

$$
\left(U_{L}\right)_{\ell i}=\sum_{n=1}^{3} \Omega_{n \ell}^{*}\left(U_{\nu}\right)_{n i}, \quad\left(U_{R}\right)_{\ell i}=\sum_{n=1}^{3} \Omega_{n \ell}^{*}\left(U_{\nu}\right)_{n+3, i},
$$

where $\Omega$ is the $3 \times 3$ unitary matrix that diagonalizes the charged lepton mass matrix $M_{\ell}$, while $U_{\nu}$ is the $6 \times 6$ unitary matrix that diagonalizes the neutrino mass matrix defined in Eq. (9). For our purposes we take the charged lepton mass matrix in diagonal form ${ }^{2}$ so that $\Omega \rightarrow 1$. In this case the overall neutrino matrix $U_{\nu}$ can be decomposed as

$$
U_{\nu}=\left(\begin{array}{c}
U_{L}^{*} \\
U_{R}
\end{array}\right)=\left(\begin{array}{ll}
U^{L L} & U^{L R} \\
U^{R L} & U^{R R}
\end{array}\right)
$$

where $U_{L}$ and $U_{R}$ relate the left-handed and right-handed neutrino flavour eigenstates $\nu_{L_{\ell}}=$ $\nu_{\ell}$ and $\nu_{R_{\ell}}=N_{\ell}$ with the mass eigenstates $\nu_{i}$,

$$
\nu_{L, R_{\ell}}=\left(U_{L, R}\right)_{\ell i} \nu_{i}, \quad i=1, \ldots, 6,
$$

and the pieces $U^{L L}, U^{L R}, U^{R L}$ and $U^{R R}$ can be calculated numerically or may be obtained in seesaw perturbation theory [16].

The charged weak interactions of the light mass eigenstate neutrinos are effectively described by the mixing matrix $U^{L L}$ which is non-unitary, hence the coupling of a given light

$\overline{2}$ This may be automatic in the presence of suitable discrete flavour symmetries as in 31 . 
neutrino to the corresponding charged lepton is decreased with respect to that of the Standard Model. This affects the rates for low energy weak decay processes, where the states that can be kinematically produced are only the light neutrinos. Similarly, right-handed neutrinos would be produced singly in the decays of the $Z$ at the CERN LEP Collider [32]. As a result there are constraints on the strength of the $U^{L R} \sim \sin \zeta_{\nu}$ mixing matrix elements that follow from all these measurements.

This formalism can be easily adapted to any seesaw model, such as the $\mathrm{SU}(2)_{L} \otimes \mathrm{SU}(2)_{R} \otimes$ $\mathrm{U}(1)_{B-L}$ scheme described in Section \A The charged current weak interactions in the leftright model can be written in terms of the neutrino mass eigenstates as,

$$
\begin{aligned}
& J_{W_{1}}^{\mu-}=\frac{g_{L}}{\sqrt{2}}\left(\bar{\nu}_{i} U_{\ell i}^{L L}+\bar{N}_{i}^{c} U_{\ell i}^{L R}\right) \gamma^{\mu} \ell_{L}+\frac{g_{R}}{\sqrt{2}} \sin \zeta_{W}\left(\bar{\nu}_{i} U_{\ell i}^{R L}+\bar{N}_{i} U_{\ell i}^{R R}\right) \gamma^{\mu} \ell_{R}, \\
& J_{W_{2}}^{\mu-}=-\frac{g_{L}}{\sqrt{2}} \sin \zeta_{W}\left(\bar{\nu}_{i} U_{\ell i}^{L L}+\bar{N}_{i} U_{\ell i}^{L R}\right) \gamma^{\mu} \ell_{L}+\frac{g_{R}}{\sqrt{2}}\left(\bar{N}_{i} U_{\ell i}^{R R}+\bar{\nu}_{i}^{c} U_{\ell i}^{R L}\right) \gamma^{\mu} \ell_{R},
\end{aligned}
$$

in analogy with Eq. (13). Here $U^{L L}, U^{R R}=\mathcal{O}(1)$ while the "small" terms are $U^{L R}, U^{R L} \sim$ $M_{D} M_{R}^{-1} \sim \sin \zeta_{\nu}$, all of which can be obtained within the seesaw perturbative diagonalization method developed in Ref. [16]. In the following, we will assume the limit in which all leftright mixing terms can be neglected, $\sin \zeta_{W}, \sin \zeta_{Z}, \sin \zeta_{\nu} \ll 1$. The applicability of this approximation for our calculations will be quantified below. As a result, the only terms surviving in (17) are

$$
\begin{aligned}
J_{W_{L}}^{\mu-} & \approx \frac{g_{L}}{\sqrt{2}} U_{\ell i} \bar{\nu}_{i} \gamma^{\mu} \ell_{L}, \\
J_{W_{R}}^{\mu-} & \approx \frac{g_{R}}{\sqrt{2}} V_{\ell i} \bar{N}_{i} \gamma^{\mu} \ell_{R},
\end{aligned}
$$

where we identified $U \equiv U_{\text {PMNS }} \equiv U^{L L}$ and $V \equiv U^{R R}$ for notational simplicity. The first term describes the mixing of light neutrinos in charged current interactions giving rise to neutrino oscillations, whereas the second term is responsible for the LHC process in Figure 1(a) as well as all low energy LFV processes as we will discuss below. We will assume manifest left-right symmetry of the gauge couplings, i.e. $g_{R}=g_{L}$, but the general case can always be recovered by simple rescaling.

\section{EXPERIMENTAL DATA}

We now turn to the existing experimental constraints on $\mathrm{SU}(2)_{L} \otimes \mathrm{SU}(2)_{R} \otimes \mathrm{U}(1)_{B-L}$ models. Experimental limits on the mass scales and mixing in the minimal LR symmetry model come from a variety of sources. For example relevant constraints can be derived from the $K_{L}-K_{S}$ mass difference, $B_{d} \bar{B}_{d}$ oscillations, $b$ quark semileptonic branching ratio and decay rate, neutrinoless double beta decay, universality tests, nonleptonic kaon decays, muon decay, lepton flavour violating processes and astrophysical constraints from nucleosynthesis and SN 1987A [33 35]. The impact of searches for neutrinoless double beta decay and lepton flavour violating processes will be discussed in more detail in Sections IIIB and IIIC, respectively. Apart from these, the most relevant constraints for our discussions are: A lower bound on the $W_{R}$ mass of $m_{W_{R}}>1.6 \mathrm{TeV}$ [36, 37] due to CP violating effects stemming from the measurement of the $K_{L}-K_{S}$ Kaon mass difference, but with uncertainties from low energy QCD corrections. A more severe limit of $m_{W_{R}}>2.5 \mathrm{TeV}$ was reported in [34, 35], 
and the authors of reference [38] argue that heavy Higgs masses lighter than $10 \mathrm{TeV}$ are disfavoured in the minimal LRSM due to low energy precision data. The minimal LRSM is therefore strongly constrained by low energy observations. At the Tevatron, searches for $W_{R} \rightarrow e \nu$ yield a limit of $m_{W_{R}}>1.12 \mathrm{TeV}$ at $95 \%$ C.L., assuming SM strength couplings [39]. Only recently, these have been superseded by limits derived from searches at the LHC for $W_{R}$ with decays into $e \nu\left(m_{W_{R}}>1.36 \mathrm{TeV}\right)$ [40] and $\mu \nu\left(m_{W_{R}}>1.40 \mathrm{TeV}\right)$ [41], again assuming SM strength couplings. Using data collected in 2010 and 2011 with an integrated luminosity of $240 \mathrm{pb}^{-1}$, the CMS collaboration has reported on the search for the production of $W_{R}$ bosons and heavy right-handed neutrinos in the minimal LRSM as in Figure 1(a). No excess has been observed, thereby excluding a region in the $\left(m_{W_{R}}, m_{N}\right)$ parameter space extending to $\left(m_{W_{R}}, m_{N}\right) \approx(1.7 \mathrm{TeV}, 0.6 \mathrm{TeV})$ [42]. Even more recently, the ATLAS collaboration has reported on a search for heavy neutrinos and right-handed $W$ bosons via the same channel with an integrated luminosity of $2.1 \mathrm{fb}^{-1}$ [43]. Again, no excess has been found in this search as well, resulting in an excluded parameter region extending to $\left(m_{W_{R}}, m_{N}\right) \approx$ $(2.5 \mathrm{TeV}, 1.5 \mathrm{TeV})$. If lighter than $\approx 5 \mathrm{GeV}$, right-handed neutrinos can be produced onshell in $B$ meson decays, and searches at LHCb provide limits on their coupling strength to muons [44, 45]. The bound on the mixing angle between $W_{R}$ and $W_{L}$ is of the order $\zeta_{W}<\mathcal{O}\left(10^{-2}\right)$ [33, 46].

Direct limits on the $Z_{R}$ mass from electroweak precision data, such as lepton universality at the $Z$ peak, are of the order $\mathcal{O}(1) \mathrm{TeV}$ [47-49]. Within the minimal LRSM one also has the theoretical relation $m_{Z_{R}} \approx 1.7 m_{W_{R}}$ for $g_{R} \approx g_{L}$, so that indirect limits via the bounds on the $W_{R}$ mass also yield more stringent constraints on the $Z_{R}$ mass. From the same data, the mixing angle between $Z_{R}$ and $Z_{L}$ is constrained to be $\zeta_{Z}<\mathcal{O}\left(10^{-4}\right)$.

\section{A. Light Neutrino Oscillation Data}

When combined with reactor and accelerator results, solar and atmospheric neutrino experiments [1-3] provide firm evidence for neutrino oscillations [4]. Together with reactor data, the experimental results on solar neutrinos clearly suggest $\nu_{e} \rightarrow \nu_{\mu, \tau}$ oscillations driven by the mass squared difference $\Delta m_{12}^{2}=m_{2}^{2}-m_{1}^{2}$ in the range of the large mixing angle solution, while the results on atmospheric neutrinos are interpreted by $\nu_{\mu} \rightarrow \nu_{\tau}$ oscillations driven by $\Delta m_{23}^{2}=m_{3}^{2}-m_{2}^{2}$ are characterized by a nearly maximal mixing. The present global analysis in a three-neutrino framework taking into account the latest results on reactor neutrino fluxes gives the following best fit values for the light neutrino squared mass differences and the mixing angles $\theta_{12}, \theta_{23}$ and $\theta_{13}$ of the neutrino mixing matrix $U$ in 
the standard parametrization [50],

$$
\begin{aligned}
\Delta m_{21}^{2} & =(7.62 \pm 0.19) 10^{-5} \mathrm{eV}^{2}, \\
\Delta m_{31}^{2} & = \begin{cases}+\left(2.53_{-0.10}^{+0.08}\right) 10^{-3} \mathrm{eV}^{2} & \mathrm{NH} \\
-\left(2.40_{-0.07}^{+0.10}\right) 10^{-3} \mathrm{eV}^{2} & \mathrm{IH},\end{cases} \\
\sin ^{2} \theta_{12} & =0.320_{-0.017}^{+0.015}, \\
\sin ^{2} \theta_{23} & = \begin{cases}0.49_{-0.05}^{+0.08} & \mathrm{NH} \\
0.53_{-0.07}^{+0.05} & \mathrm{IH},\end{cases} \\
\sin ^{2} \theta_{13} & = \begin{cases}0.026_{-0.004}^{+0.003} & \mathrm{NH} \\
0.027_{-0.004}^{+0.003} & \mathrm{IH},\end{cases}
\end{aligned}
$$

for normal (NH) and inverse hierarchy (IH), respectively.

\section{B. Neutrinoless Double Beta Decay}

The most sensitive probe of the absolute mass scale of the neutrinos is neutrinoless double $\beta$ decay $(0 \nu \beta \beta)$. In this process, an atomic nucleus with $Z$ protons decays into a nucleus with $Z+2$ protons and the same mass number $A$ under the emission of two electrons,

$$
(A, Z) \rightarrow(A, Z+2)+2 e^{-} .
$$

This process can be engendered through the exchange of a light neutrino connecting two V-A weak interactions, as illustrated in Figure 2(a). The process in Eq. (20) is lepton number violating and, in the standard picture of light neutrino exchange, it is only possible if the neutrino is identical to its own anti-particle, i.e. if neutrinos are Majorana particles. In fact quite generally one may argue that, whatever the underlying mechanism inducing $0 \nu \beta \beta$ decay, its observation implies the Majorana nature of neutrinos [8, 9].

Currently, the best limit on $0 \nu \beta \beta$ decay comes from the search for $0 \nu \beta \beta$ decay of the isotope ${ }^{76} \mathrm{Ge}$ giving a half-life of $T_{1 / 2}>1.9 \cdot 10^{25}$ yrs [10]. This results in an upper bound on the effective $0 \nu \beta \beta$ Majorana neutrino mass of $m_{\beta \beta} \equiv\left|\sum_{i} U_{e i}^{2} m_{\nu_{i}}\right|<300-600 \mathrm{meV}$, depending on the model used to calculate the nuclear matrix element of the process. A controversial claim of observation of $0 \nu \beta \beta$ decay in ${ }^{76} \mathrm{Ge}$ gives a half-life of $T_{1 / 2}=(0.8-$ 18.3) $\cdot 10^{25}$ yrs [51] and a resulting effective Majorana neutrino mass of $m_{\beta \beta}=110-560 \mathrm{meV}$. Next generation experiments such as SuperNEMO, GERDA, CUORE, EXO or MAJORANA aim to increase the half-life sensitivity by one order of magnitude and will confirm or exclude the claimed observation. The planned experiment SuperNEMO allows the measurement of $0 \nu \beta \beta$ decay in several isotopes to the ground and excited states and is able to track the trajectories of the emitted electrons and determine their individual energies. In this respect, the SuperNEMO experiment has a unique potential to disentangle the possible mechanisms for $0 \nu \beta \beta$ decay [52, 53].

In the left-right symmetric model, several such mechanisms can contribute to $0 \nu \beta \beta$ as shown in Figure 2, Here, contributions (a)-(d) are due to the exchange of either light or heavy neutrinos as well as light and heavy $W$ bosons. All these terms could be described by a single Feynman diagram using the mass eigenstates $n_{i}(i=1, \ldots, 6)$ and $W_{a}(a=1,2)$ of neutrinos and $W$ bosons. The separation into the four contributions shown in Figure 2 is traditionally 


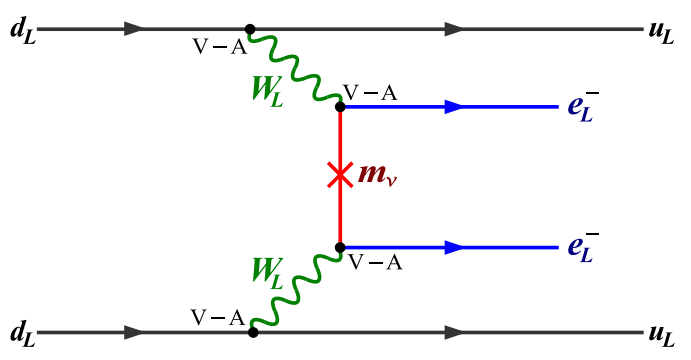

(a)

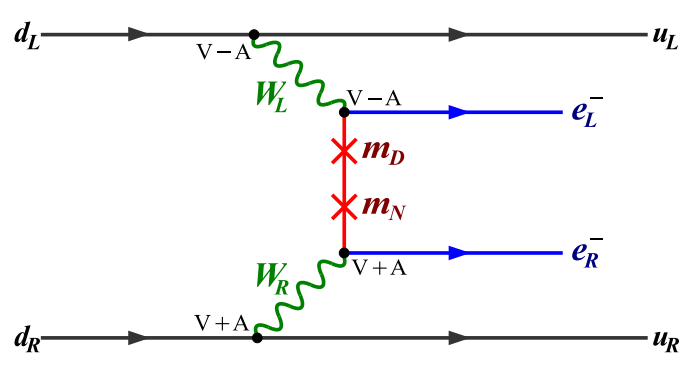

(c)

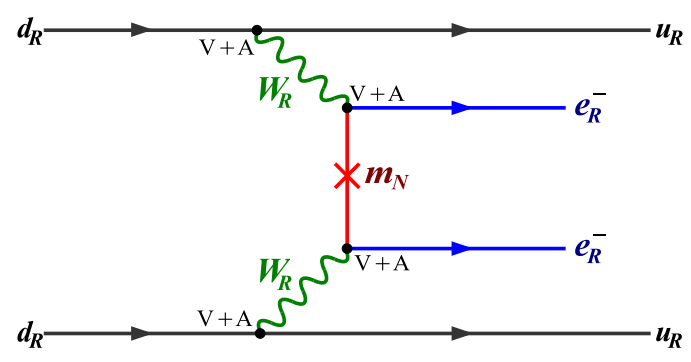

(b)

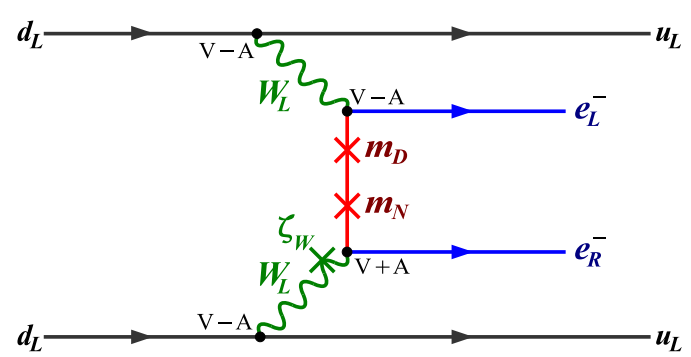

(d)

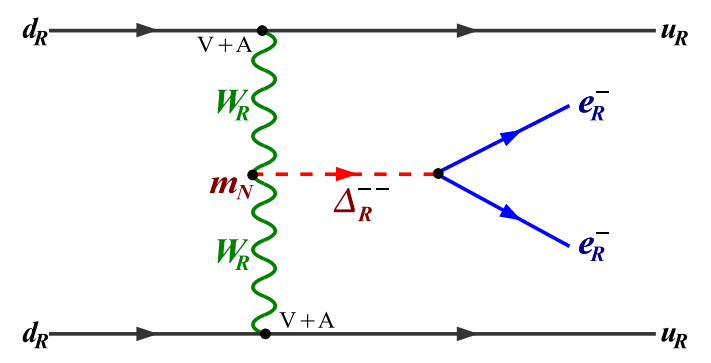

(e)

FIG. 2: Diagrams contributing to $0 \nu \beta \beta$ decay in left-right symmetry: (a) Light neutrino exchange (standard mass mechanism), (b) Heavy neutrino exchange, (c) Neutrino and heavy $W$ exchange with Dirac mass helicity flip ( $\lambda$ mechanism), (d) Neutrino and light $W$ exchange with Dirac mass and $W$ mixing suppression ( $\eta$ mechanism), (e) Doubly charged Higgs Triplet exchange.

used as it illustrates the dependence on and suppression with the different LRSM model parameters. Diagram 2(a) describes the exchange of massive light neutrinos corresponding to the generally considered mass mechanism. Its contribution to $0 \nu \beta \beta$ depends on the effective neutrino mass $m_{\beta \beta}=\left|\sum_{i} U_{e i}^{2} m_{\nu_{i}}\right|$, and saturates current experimental bounds if the light neutrinos are degenerate with mass scale $m_{\nu_{1}} \approx m_{\beta \beta} \approx 0.3-0.6 \mathrm{eV}$.

Correspondingly, diagram 2(b) describes the exchange of heavy right-handed neutrinos 


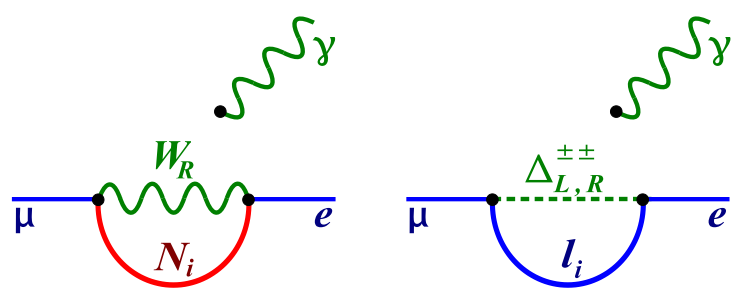

FIG. 3: Diagrams contributing to $\mu \rightarrow e \gamma$. To form complete diagrams, the external photon can be attached to any charged particle line.

and depends on the effective coupling

$$
\epsilon_{N}=\sum_{i=1}^{3} V_{e i}^{2} \frac{m_{p}}{m_{N_{i}}} \frac{m_{W_{L}}^{4}}{m_{W_{R}}^{4}} .
$$

If this is the dominant contribution to $0 \nu \beta \beta$, current experimental limits correspond to $\left|\epsilon_{N}\right| \lesssim 2 \times 10^{-8}[54]$.

Diagrams 2(c) and (d) are suppressed by the left-right mixing $M_{D} / M_{N} \sim \zeta_{\nu} \sim \sqrt{m_{\nu} / m_{N}}$ (the latter relation is valid for a dominant type-I seesaw mass mechanism [16]) between light and heavy neutrinos. In our approach we assume that this mixing is small enough so that decays of heavy neutrinos via this Yukawa coupling are negligible compared to the three-body decays via the $S U(2)_{R}$ gauge coupling. In this case, these contributions to $0 \nu \beta \beta$ are also generally negligible, and we will not discuss them further here.

Diagram 2(e) describes the contribution from the exchange of a right-handed doublycharged triplet Higgs $\Delta_{R}^{--3}$, with the effective coupling

$$
\epsilon_{\Delta}=\sum_{i=1}^{3} V_{e i}^{2} \frac{m_{N_{i}} m_{p}}{m_{\Delta_{R}^{-}}^{2}} \frac{m_{W_{L}}^{4}}{m_{W_{R}}^{4}} .
$$

If dominant, current experimental limits correspond to $\left|\epsilon_{\Delta}\right| \lesssim 8 \times 10^{-8}[\underline{54}]$.

\section{Low Energy Lepton Flavour Violating Processes}

The existence of neutrino oscillations suggests that, at some level, lepton flavour violation should also take place in other processes. When taking into account only light neutrinos, LFV is strongly suppressed by $\left(\Delta m_{\nu}^{2} / m_{W}^{2}\right) \approx 10^{-50}$, due to the GIM mechanism. This

\footnotetext{
${ }^{3}$ A priori, there is an analogous diagram with a left-handed doubly-charged Higgs, but its contribution is always sub-dominant to the standard mass mechanism unless there is a fine-tuning between type-I and type-II seesaw contributions to the light neutrino masses.
} 

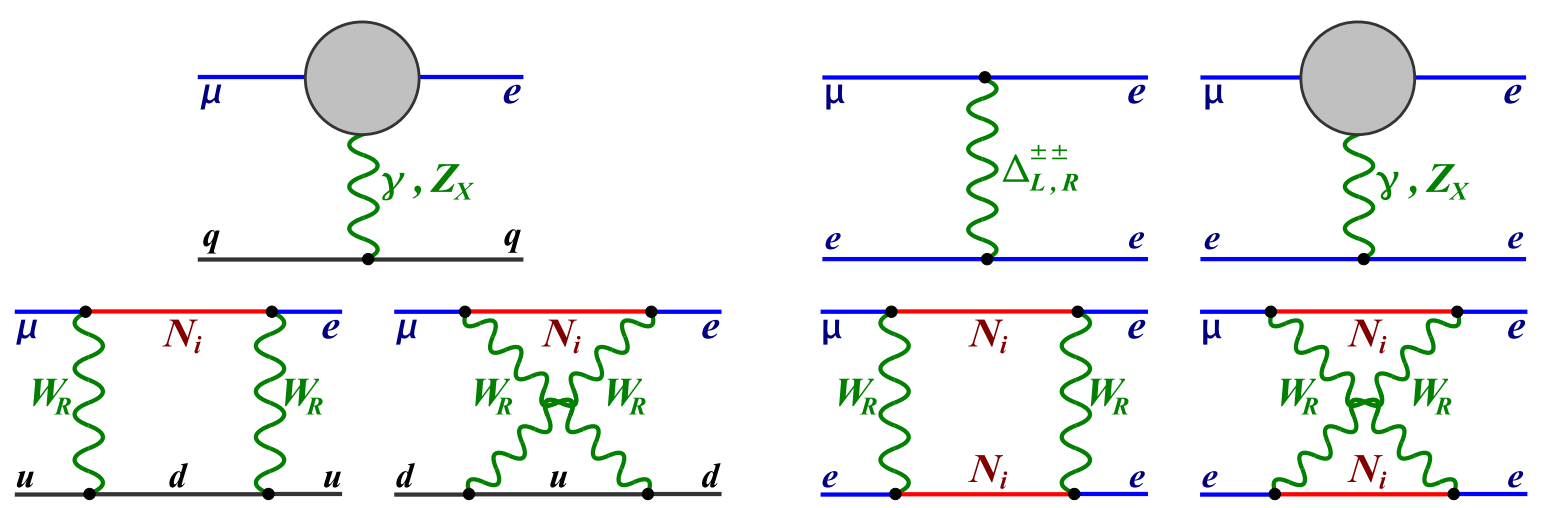

FIG. 4: Diagrams contributing to $\mu \rightarrow e$ conversion in nuclei (left) and $\mu \rightarrow e e e$ (right) in left-right symmetry. The grey circle represents the effective $\mu-e$-gauge boson vertex with contributions from Figure 3 ,

results in LFV process rates far below any experimental sensitivity which can be safely ignored. Within the LRSM, charged lepton flavour violation naturally occurs due to potentially large flavour violating couplings of the heavy right-handed neutrinos and Higgs scalars with charged leptons. Amongst a wide range of possible low energy LFV observables, these give rise to observable rates for the processes $\mu \rightarrow e \gamma, \mu \rightarrow$ eee and $\mu \rightarrow e$ conversion in nuclei, cf. Figures 3 and 4 .

Taking into account contributions from heavy right-handed neutrinos and Higgs scalars, the expected branching ratios and conversion rates of the above processes have been calculated in the LRSM in [55]. In general, these depend on many parameters, but under the assumption of similar mass scales between the heavy particles in the LRSM, $m_{N_{i}} \approx m_{W_{R}} \approx m_{\Delta_{L}^{--}} \approx m_{\Delta_{R}^{--}}$one can make simple approximations. Such a spectrum is naturally expected, as all masses are generated in the breaking of the right-handed symmetry. Under this assumption, the expected branching ratios are given by [55]

$$
\begin{aligned}
B r(\mu \rightarrow e \gamma) & \equiv \frac{\Gamma\left(\mu^{+} \rightarrow e^{+} \gamma\right)}{\Gamma\left(\mu^{+} \rightarrow e^{+} \nu \bar{\nu}\right)} \\
& \approx 1.5 \times 10^{-7}\left|g_{e \mu}\right|^{2}\left(\frac{1 \mathrm{TeV}}{m_{W_{R}}}\right)^{4} \\
R^{N}(\mu \rightarrow e) & \equiv \frac{\Gamma\left(\mu^{-}+{ }_{Z}^{A} N \rightarrow e^{-}+{ }_{Z}^{A} N\right)}{\Gamma\left(\mu^{-}+{ }_{Z}^{A} N \rightarrow \nu_{\mu}+{ }_{Z-1}^{A} N^{\prime}\right)} \\
& \approx X_{N} \times 10^{-7}\left|g_{e \mu}\right|^{2}\left(\frac{1 \mathrm{TeV}}{m_{\Delta_{R}^{--}}}\right)^{4} \alpha\left(\log \frac{m_{\Delta_{R}^{-}}^{2}}{m_{\mu}^{2}}\right)^{2} \\
\operatorname{Br}(\mu \rightarrow e e e) & \equiv \frac{\Gamma\left(\mu^{+} \rightarrow e^{+} e^{-} e^{+}\right)}{\Gamma\left(\mu^{+} \rightarrow e^{+} \nu \bar{\nu}\right)} \\
& \approx \frac{1}{2}\left|h_{e \mu} h_{e e}^{*}\right|^{2}\left(\frac{m_{W_{L}}^{4}}{m_{\Delta_{R}^{-}}^{4}}+\frac{m_{W_{L}}^{4}}{m_{\Delta_{L}^{-}}^{4}}\right)
\end{aligned}
$$

Here, $X_{(\mathrm{Al}, \mathrm{Ti}, \mathrm{Au})} \approx(0.8,1.3,1.6)$ is a nucleus-dependent factor whereas $g_{e \mu}$ and $h_{i j}$ describe the effective lepton-gauge boson couplings and lepton-Higgs coupling in (quasi-)manifest 
left-right symmetry,

$$
\begin{aligned}
g_{e \mu} & =\sum_{n=1}^{3} V_{e n}^{*} V_{\mu n}\left(\frac{m_{N_{n}}}{m_{W_{R}}}\right)^{2}, \\
h_{i j} & =\sum_{n=1}^{3} V_{i n} V_{j n}\left(\frac{m_{N_{n}}}{m_{W_{R}}}\right), \quad i, j=e, \mu, \tau .
\end{aligned}
$$

As shown in [55], the above approximations are valid if the masses generated in breaking the right-handed symmetry are of the same order with $0.2 \lesssim m_{i} / m_{j} \lesssim 5$ for any pair of $m_{i, j}=m_{N_{n}}, m_{W_{R}}, m_{\Delta_{L}^{--}}, m_{\Delta_{R}^{--}}$. In our approach, we keep $m_{W_{R}}$ and $m_{N_{n}}$ as free parameters of the order of $0.5-5 \mathrm{TeV}$, relevant for LHC searches, and consider an order of magnitude variation in the heavy Higgs masses of $0.3<m_{\Delta_{L, R}^{--}} / m_{W_{R}}<3$.

Several properties of the Eqs. (23)-(25) can then be derived: (i) Both $\operatorname{Br}(\mu \rightarrow e \gamma)$ and $R^{N}(\mu \rightarrow e)$ are proportional to the LFV factor $\left|g_{e \mu}\right|^{2}$. In addition, as $\alpha \log \left((5 \mathrm{TeV})^{2} / m_{\mu}^{2}\right)=$ $\mathcal{O}(1)$, the ratio of their rates gives $R^{N}(\mu \rightarrow e) / \operatorname{Br}(\mu \rightarrow e \gamma)=\mathcal{O}(1)$, independent of the right-handed neutrino mixing matrix $V$ and largely independent of the heavy particle spectrum. This consequence of the logarithmic enhancement of the doubly-charged Higgs boson contributions to $\mu \rightarrow e$ conversion is in stark contrast to models where the symmetry breaking occurs far above the electroweak scale, such as in supersymmetric seesaw models with low $\tan \beta$. Here, the $Z$ and photon penguin contributions dominate, and $R^{N}(\mu \rightarrow e) / \operatorname{Br}(\mu \rightarrow e \gamma) \propto \alpha \lesssim \mathcal{O}\left(10^{-2}\right)$. (ii) Unless there are cancellations, the LFV couplings are $\left|g_{e \mu}\right| \approx\left|h_{e e}^{*} h_{e \mu}\right|$ and therefore $\operatorname{Br}(\mu \rightarrow e e e) / R^{N}(\mu \rightarrow e)=\mathcal{O}(300)$ (for $m_{\Delta_{L, R}^{--}} \approx 1$ $\mathrm{TeV})$.

The above theoretical predictions are to be compared with the current experimental upper limits at $90 \%$ C.L. [56 58],

$$
\begin{aligned}
B r_{\exp }(\mu \rightarrow e \gamma) & <2.4 \cdot 10^{-12}, \\
R_{\exp }^{A u}(\mu \rightarrow e) & <8.0 \cdot 10^{-13}, \\
B r_{\exp }(\mu \rightarrow e e e) & <1.0 \cdot 10^{-12}
\end{aligned}
$$

With the current experimental limits roughly of the same order, it follows that the most restrictive parameter bounds in the LRSM are derived from the limits on $\operatorname{Br}(\mu \rightarrow e e e)$.

As for future developments, the currently running MEG experiment [56] aims for a sensitivity of

$$
\operatorname{Br}_{\mathrm{MEG}}(\mu \rightarrow e \gamma) \approx 10^{-13}
$$

whereas the COMET and Mu2e experiments both plan to reach a sensitivity of [59, 60]

$$
R_{\mathrm{COMET}}^{A l}(\mu \rightarrow e) \approx 10^{-16}
$$

\section{DILEPTON SIGNALS AT THE LHC}

In the following we discuss the LHC potential to discover lepton flavour and lepton number violating dilepton signals from the production of a heavy right-handed neutrino,

$$
p+p \rightarrow W_{R}^{ \pm} \rightarrow \ell_{a}^{ \pm}+N_{R}
$$




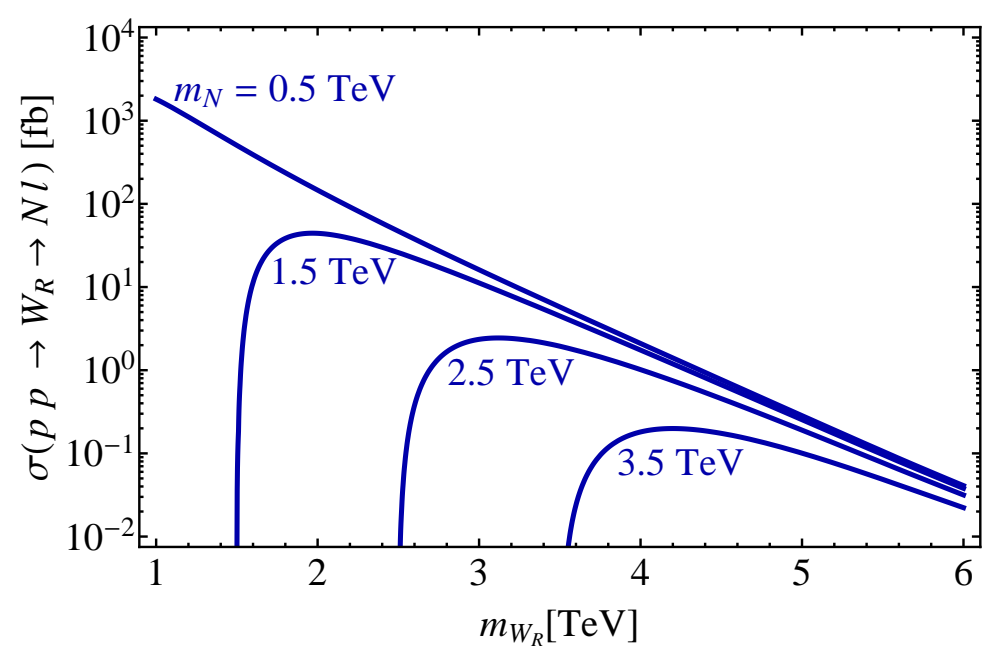

FIG. 5: Total cross section of the process $p p \rightarrow W_{R} \rightarrow N_{R} \ell$ as a function of the $W_{R}$ mass, for different values of the neutrino $N_{R}$ mass, calculated with PROTOS 2.1 [66] for the LHC with $\sqrt{s}=14 \mathrm{TeV}$.

followed by a three-body decay of $N_{R}$, as shown in Figure 1(a),

$$
N_{R} \rightarrow \ell_{b}^{\mp}+W_{R}^{*} \rightarrow \ell_{b}^{\mp}+2 j .
$$

This process is the main production channel for the right handed neutrinos $N_{R}$, where the cross section is typically enhanced, compared to other production mechanisms, via on-shell $Z_{R}$ production (Figure 1(b)) and $W_{R}$ fusion (Figure 1(c)) 61 64].

Since the signal has no missing energy, and since the heavy on-shell $W_{R}$ subsequently decays into an on-shell $N_{R}$, backgrounds can be removed and the signal well identified by the two $W_{R}$ and $N_{R}$ resonances in the respective invariant mass spectra, see Figure 7. The dilepton signals in left-right symmetric models have already been studied [63 [65], and bounds on the $W_{R}$ and $N_{R}$ masses have been obtained [41, 43].

Here we extend the existing analyses and include lepton flavour violation in the righthanded neutrino sector to assess the LHC potential to probe the right-handed neutrino sector. This allows us not only to determine the $W_{R}$ and $N_{R}$ masses, but also to unravel the flavour mixing pattern in the heavy $N_{R}$ sector. These results will be confronted with low energy probes of rare LFV processes and neutrinoless double beta decay.

\section{A. Event Simulation, Topology and Cut Flow}

We generate the partonic signal events $q \bar{q} \rightarrow W_{R} \rightarrow \ell_{a} N_{R}$, followed by the threebody decay $N_{R} \rightarrow \ell_{b} j j$, with the Triada 1.1 generator [67] of the Monte Carlo package PROTOS 2.1 [66]. As a benchmark scenario we choose

$$
m_{W_{R}}=2 \mathrm{TeV}, \quad m_{N_{R}}=0.5 \mathrm{TeV}
$$

where we assume the equality of the two $\mathrm{SU}(2)$ gauge couplings for all calculations, $g_{R}=g_{L}$, and only one of the three neutrinos $N_{R}$ is lighter than the $W_{R}$ boson. If other neutrino 


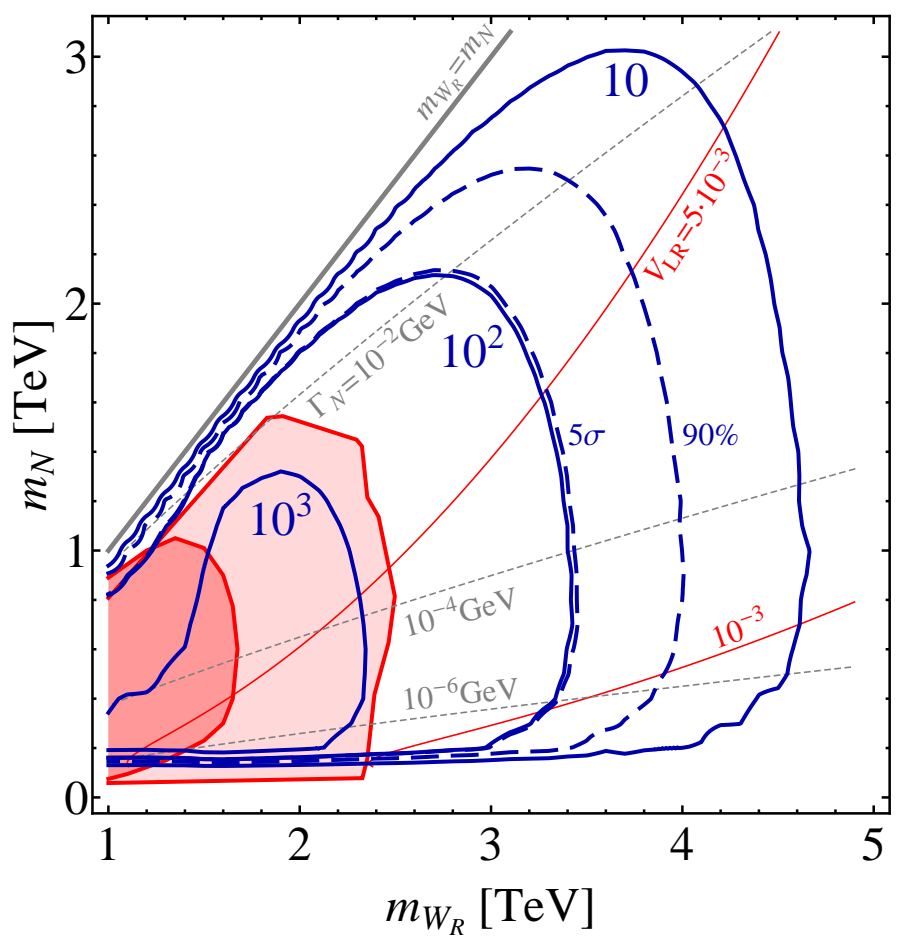

FIG. 6: Discovery reach of the LHC with $\sqrt{s}=14 \mathrm{TeV}$ and $\mathcal{L}=30 \mathrm{fb}^{-1}$ in the $m_{W_{R}}-m_{N}$ parameter plane. The blue solid contours denote constant event rates of the signal process $p p \rightarrow W_{R} \rightarrow$ $\ell \ell+2$ jets, after event reconstruction and selection cuts (see Section IVA3). The blue dashed contours indicate the discovery reach with $S / \sqrt{B}=5$, and the exclusion region at the $90 \%$ C.L. The grey solid contour indicates the kinematical threshold $m_{W_{R}}=m_{N}$ for the on-shell decay $W_{R} \rightarrow \ell N_{R}$. The grey dashed lines are contours of constant neutrino width $\Gamma_{N}$. Above the red solid contours the neutrino decays via an off-shell $W_{R}$ start to dominate, i.e. $\operatorname{Br}\left(N \rightarrow \ell W_{R}^{*}\right)>0.5$, over the SM decay modes $N \rightarrow \ell W, \nu Z, \nu H$ [68] for a given value of $V_{L R}^{2}=\sin ^{2} \zeta_{W}+\sin ^{2} \zeta_{\nu}$, see Eq. (13). The red shaded areas are excluded by current LHC searches at CMS [42] (dark shaded) and ATLAS [43] (light shaded).

states are lighter than $W_{R}$, and if their mass difference is sufficiently large, their signals can be separated by the resonances in the invariant mass distributions, see Figure 7 . Due to QCD radiation, the small inherent widths of the neutrinos of some $10 \mathrm{keV}$ get broadened up to $\approx 50 \mathrm{GeV}$. As discussed above, we assume negligible left-right mixing, with $\zeta_{W}, \zeta_{Z}$ and $U^{L R} \sim \zeta_{\nu}$ smaller than $10^{-4}$ [68], such that the SM decays $N_{R} \rightarrow W \ell, Z \nu, H \nu$ are sufficiently suppressed ${ }^{4}$. For the decays of the heavy $W_{R}$ boson and the neutrino we obtain the branching ratios $\operatorname{Br}\left(N_{R} \rightarrow \ell q q^{\prime}\right) \approx 83 \%$, for $q=u, c, q^{\prime}=d, s, \operatorname{Br}\left(N_{R} \rightarrow \ell t b\right) \approx 17 \%$, and $\operatorname{Br}\left(W_{R} \rightarrow \ell N_{R}\right) \approx 9 \%$ in our benchmark scenario.

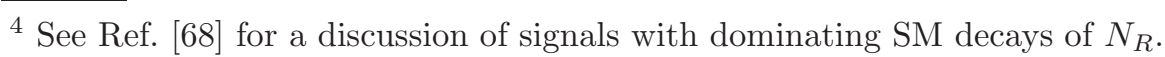




\section{Signal Topology}

In Figure 5, we show the $m_{W_{R}}$ dependence of the leading order total production cross section $\sigma\left(p p \rightarrow W_{R} \rightarrow \ell \ell+2 j\right)$. For $m_{W_{R}} \approx 2.5 \mathrm{TeV}$ and $m_{N} \lesssim 1.5 \mathrm{TeV}$ (the exclusion reach of current LHC searches), cross sections up to 30 - $50 \mathrm{fb}$ are possible. In Figure 6, we show the expected event rates after reconstruction and cuts (as described below) in the $m_{W_{R}}-m_{N_{R}}$ plane. To understand the main kinematic features in the production and decay of $W_{R}$ and $N_{R}$, the parameter region can be divided into three different kinematic areas:

1) The threshold region, with $m_{N_{R}} \lesssim m_{W_{R}}$, is close to where the two-body decay $W_{R} \rightarrow$ $\ell N_{R}$ is kinematically forbidden, and the signal cross section and thus the LHC sensitivity are therefore suppressed.

2) The jet region, with $m_{N_{R}} \ll m_{W_{R}}$. Due to the large mass difference, the $N_{R}$ is highly boosted. The lepton from the subsequent decay $N_{R} \rightarrow \ell+2 j$ is not isolated but tends to lie inside the cone of the two jets. Although the bare signal cross section is almost constant for fixed $m_{W_{R}}$, the LHC sensitivity in this region is quickly suppressed for $m_{N} \lesssim 200 \mathrm{GeV}$.

3) The discovery reach zone, with $m_{N_{R}}<m_{W_{R}}$ enables ideal decay kinematics with a large production cross section and isolated leptons such that the best bounds on the $W_{R}$ mass can be set.

The blue contours in Figure 6 give an overview of the LHC reach to probe the $W_{R}$ and $m_{N_{R}}$ masses. As shown by the red contours in Figure 6, the value of the mixing parameter $V_{L R}^{2}=\sin ^{2} \zeta_{W}+\sin ^{2} \zeta_{\nu}$, see Eq. (13) with $g_{L}=g_{R}$, must be chosen sufficiently small, $V_{L R} \lesssim 10^{-3}$ to suppress the SM decays $N \rightarrow \ell W, \nu Z, \nu H$ in the relevant parameter space, which would otherwise lead to trilepton signatures, see Ref. [68].

\section{Background Events}

Since our signal consists of two isolated leptons and at least two high $p_{T}$ jets, the major SM background sources for opposite-sign dileptons stem from $Z+j(j)$ (one or two hard jets) and $t \bar{t}$ production. These backgrounds have total cross sections of the order of $2 \times$ $10^{3} \mathrm{pb}(Z+j(j))$ and $5 \times 10^{2} \mathrm{pb}(t \bar{t})$, compared to the maximally possible signal cross section of $\approx 5 \times 10^{-2} \mathrm{pb}$. Other SM backgrounds with subdominant cross sections arise from diboson production, $p p \rightarrow W W, W Z, Z Z, W H, Z H$. Note that these SM background sources only provide opposite-sign dileptons, whereas same-sign dilepton pairs may originate from charge mis-identification, mistakenly reconstructed leptons from jets and SM diboson production [69], which is generally small compared to opposite-sign background. We use the code Alpgen [70] to generate the SM $Z$ +jets background, requiring a minimal jet transverse momentum of $p_{T}>20 \mathrm{GeV}$ to reduce $\mathrm{CPU}$ time, and later using proper matching to take into account final state radiation. The parton shower Monte Carlo Pythia 6.4 [71] is used to generate the SM backgrounds $p p \rightarrow t \bar{t}$ at leading order. For $t \bar{t}$, we take NLO effects into account by multiplication with a factor $\kappa=1.6$. All hard partonic events are then passed to Pythia, to add initial and final state radiation and pile-up, and to perform hadronization. Proper matching is applied for initial and final state radiation for the $Z+$ jets Alpgen events. 


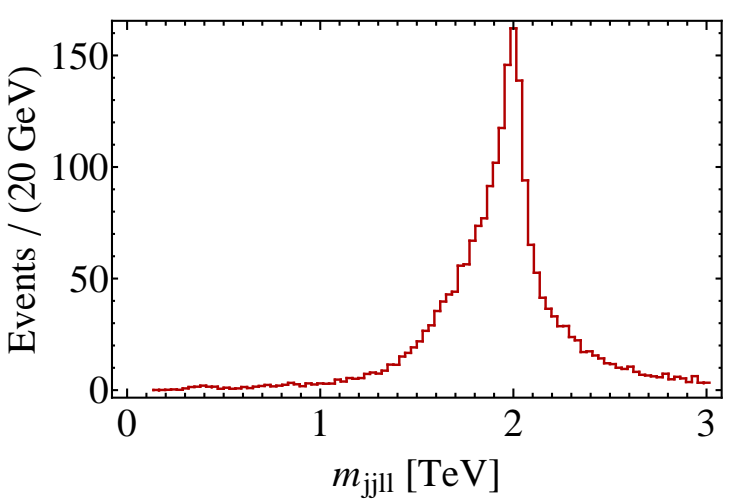

(a)

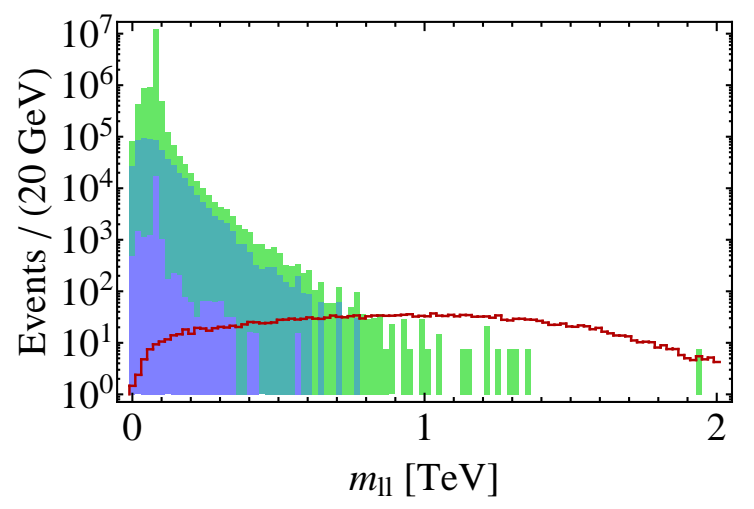

(c)

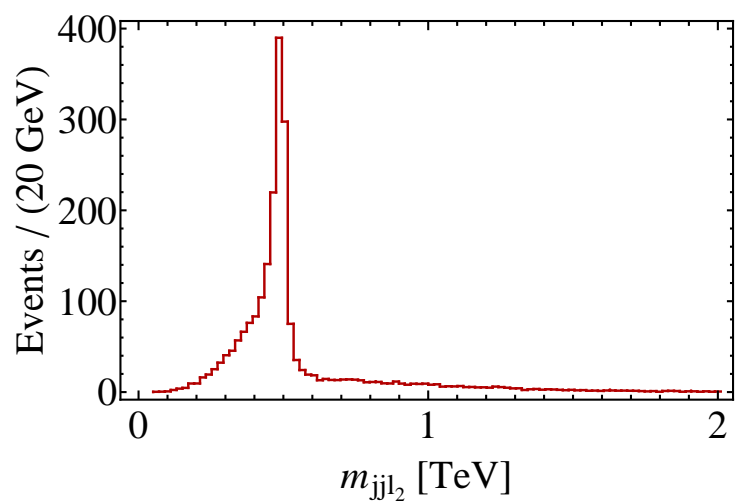

(b)

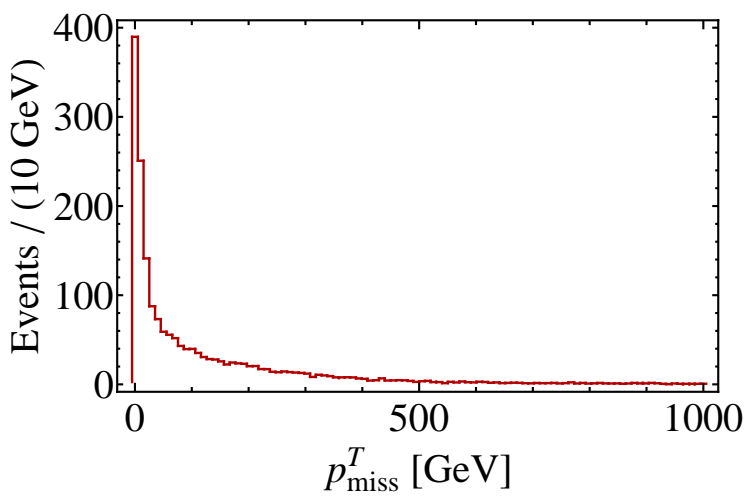

(d)

FIG. 7: Distribution of signal events (red) in the invariant masses of the particle combinations $j j \ell_{1} \ell_{2}$ (a), $j j \ell_{2}$ (b), $\ell_{1} \ell_{2}$ (c), as well as the missing transverse momentum (d) in the benchmark scenario Eq. (33), at the $\mathrm{LHC}$ with $14 \mathrm{TeV}$ and $\mathcal{L}=30 \mathrm{fb}^{-1}$. We denote with $\ell_{1(2)}$ the leptons with the highest (second highest) $p_{T}$. In (c), stacked background events are shown for $Z+$ jets (light green), $t \bar{t}$ (dark green), and $W+Z$ (blue) production.

\begin{tabular}{cc}
\hline number of jets & $N_{j} \geq 2$ \\
number of isolated leptons & $N_{\ell}=2$ \\
invariant dilepton mass & $m_{\ell \ell}>0.3 \mathrm{TeV}$ \\
total invariant mass & $m_{\ell \ell j j}>1.5 \mathrm{TeV}$ \\
\hline
\end{tabular}

TABLE I: Selection cuts used in the LHC event analysis.

\section{Detector Simulation, Event Reconstruction and Selection Cuts}

To simulate a generic LHC detector, we use the fast detector simulation package AcerDET1.0 [72] with standard settings, for the simulated signal and background events. As we are 


\begin{tabular}{r|rr|rr|rr|rrrr} 
& \multicolumn{7}{|c|}{ OS } & \multicolumn{7}{|c}{ SS } \\
& $e^{+} e^{-} \mu^{+} \mu^{-}$ & $e^{+} \mu^{-} e^{-} \mu^{+}$ & $e^{+} \mu^{+}$ & $e^{-} \mu^{-}$ & $e^{+} e^{+}$ & $e^{-} e^{-} \mu^{+} \mu^{+}$ & $\mu^{-} \mu^{-}$ \\
\hline$t \bar{t}$ & 190 & 170 & 149 & 164 & $\approx 10$ & $\approx 10$ & $\approx 10$ & $\approx 10$ & $\approx 10$ & $\approx 10$ \\
$Z j(j)$ & 181 & 187 & 0 & 2 & 0 & 0 & $\approx 10$ & $\approx 10$ & $\approx 10$ & $\approx 10$ \\
\hline Signal & 289 & 192 & 228 & 230 & 330 & 108 & 204 & 74 & 146 & 45 \\
Eff. [\%] & 51 & 33 & 42 & 43 & 41 & 41 & 49 & 50 & 35 & 32
\end{tabular}

TABLE II: Background and signal events after cuts, Table I for our benchmark scenario, Eq. (33), with maximal mixing to electrons and muons, $V_{N e}=V_{N \mu}=1 / \sqrt{2}, V_{N \tau}=0$, at the LHC with $14 \mathrm{TeV}$ and $\mathcal{L}=30 \mathrm{fb}^{-1}$. The dileptons are grouped into pairs with same-sign (SS) and oppositesign (OS) charges. The background event rates for the same-sign signatures were estimated using a $5 \%$ probability of mis-identifying the charge of one of the leptons. For the signal, we give the efficiencies (Eff.) as ratios of events after and before reconstruction and selection cuts in percent.

interested in lepton flavour violating signals, we generate the event sample using a maximal mixing of the active right-handed neutrino to both electrons and muons, but without coupling to taus, i.e. we have $V_{N e}=V_{N \mu}=1 / \sqrt{2}$ and $V_{N \tau}=0$. For the primary selection, we require two AcerDET reconstructed isolated leptons $(\ell=e, \mu)$ and at least two jets. We show the invariant mass distributions of signal and background events before applying cuts in Figure 7 .

We then apply our selection cuts as summarized in Table凹, to reduce the background [63, 64. Dilepton pairs from $Z+$ jets events are efficiently reduced by requiring a large dilepton mass $m_{\ell \ell}>300 \mathrm{GeV}$. A cut well above the $Z$-peak is necessary, due to the long tail in the $Z \rightarrow \ell \ell$ invariant mass distribution, see Figure 17. In addition, a generous cut on the total invariant mass $m_{\ell \ell j j}>1.5 \mathrm{TeV}$ reduces $t \bar{t}$ and $Z$ +jets further, without reducing the signal too much, which peaks at $m_{\ell \ell j j} \approx m_{W_{R}}$, typically with a width of order $100 \mathrm{GeV}$ due to final gluon radiation and smearing. In Figure 7, we also show the signal distribution with respect to the missing transverse momentum, but only for illustration, as a cut on this variable does not provide a noticeable improvement of the signal over background ratio. Nevertheless, appropriate selection criteria with respect to the missing momentum can be useful, e.g. in order to decrease possible containment of LNV and LFV signals with SM background processes containing light neutrinos.

For our benchmark scenario, we summarize the background and signal events after cuts in Table II. With these cuts, we obtain signal efficiencies of $\epsilon \approx 50 \%, 43 \%, 34 \%$ for the dilepton flavour compositions $\ell \ell^{\prime}=e e, e \mu, \mu \mu$, respectively. Note that typically the AcerDET reconstruction efficiency is higher for electrons than muons. In addition, the AcerDET algorithms are not including inefficiencies for the $e$ and $\mu$ reconstruction. Additional weighting factors of $70-90 \%$ for each lepton could be applied. On the other hand, we do not include a factor $k \approx 1.3$ for the signal events [73]. In Table II] we have also grouped the dileptons into pairs with same-sign (SS) and opposite-sign (OS) charges. The background rates of the same-sign signatures were estimated using a $5 \%$ probability of mis-identifying the charge of one of the leptons in the respective opposite-sign signature [63]. No other sources for SS background were taken into account. For $W_{R}$ masses of the order of $\mathrm{TeV}$, the valence quarks play a significant role for its production, and thus, reflected by the PDFs, the rate for $W_{R}^{+}$production is larger than that for $W_{R}^{-}[63]$. Typically the fraction $W_{R}^{+} /\left(W_{R}^{+}+W_{R}^{-}\right)$ 
of produced $W_{R}^{+}$in $p p$ collisions at $14 \mathrm{TeV}$ changes from $\approx 70 \%$ to $\approx 95 \%$ for $m_{W_{R}}$ increasing from $1 \mathrm{TeV}$ to $10 \mathrm{TeV}$ [63, 64]. We thus obtain for the reconstructed signal events $N\left(e^{+} e^{-}\right): N\left(e^{+} e^{+}\right): N\left(e^{-} e^{-}\right) \approx N\left(\mu^{+} \mu^{-}\right): N\left(\mu^{+} \mu^{+}\right): N\left(\mu^{-} \mu^{-}\right) \approx N\left(e^{+} \mu^{-}+e^{-} \mu^{+}\right):$ $N\left(e^{+} \mu^{+}\right): N\left(e^{-} \mu^{-}\right) \approx 4: 3: 1$, in our benchmark scenario.

\section{Selection Cut Optimization}

For the purpose of our study, we do not optimize the cuts for each LRSM parameter point defined by $m_{W_{R}}$ and $m_{N_{R}}$. Instead, we always use the cuts as given in Table \, which were chosen to highlight the discovery reach for the LHC. The cuts could be adapted by requiring higher transverse momenta of the leptons and jets, $p_{\mathrm{T}} \gtrsim 100 \mathrm{GeV}$ as well as selecting on missing $p_{\mathrm{T}}^{\text {miss }}<50 \mathrm{GeV}$ [68]. Further, one can apply mass-window cuts to isolate the $W_{R}$ and $N_{R}$ mass peaks in the $m_{\ell j j}$ and $m_{\ell \ell j j}$ invariant mass distributions, respectively [63, 64]. In particular, different $m_{\ell j j}$ mass-window cuts might be necessary to disentangle different signal contributions, if more than one $N_{R}$ is kinematically accessible. Also, our cut on the total invariant mass $m_{\ell \ell j j}>1.5 \mathrm{TeV}$ is chosen for heavy bosons $m_{W_{R}} \gtrsim 2 \mathrm{TeV}$, and for smaller masses one loses signal events, which can be observed in the indentation on the left side of the $10^{3}$ events contour in Figure 6 ,

The cuts might also be improved in the kinematically suppressed parameter regions, i.e. for $m_{N_{R}} \lesssim m_{W_{R}}$ (threshold region) and $m_{N_{R}} \ll m_{W_{R}}$ (jet region). To enhance the sensitivity in the threshold region, same-sign lepton pairs can be selected, or the cuts can be raised for the $p_{T}$ of the jets and for the dilepton invariant mass $m_{\ell \ell}$ [63]. In the jet region, where the lepton from the $N_{R}$ decay tends to lie inside the jet cones, one could search for events with one high $p_{T}$ isolated lepton and one high $p_{T}$ hadronic jet with a large electromagnetic component, and matching a high $p_{T}$ track in the inner detector. This typically increases the sensitivity in those regions by a few percent [63, 64].

For same-sign dileptons, the kinematic cuts can be relaxed, since SM background events will dominantly produce opposite-sign dileptons. As mentioned above, same-sign lepton background events would originate from charge mis-identification, mistakenly reconstructed leptons from jets and SM diboson production [69]. The dilepton pairs could also be grouped according to their flavour configurations; for example, lepton pairs with different flavours only originate from $t \bar{t}$ production, reducible by vetoing $b$-jets. Since the b-tagging efficiencies at the LHC are of the order of $60 \%$ to $70 \%$, the $t \bar{t}$ background should be reduced by an order of magnitude. The signal loss will be small, since we typically have $\operatorname{Br}\left(N_{R} \rightarrow \ell b t\right) \approx 20 \%$, and $\operatorname{Br}(t \rightarrow b \ell \nu)=20 \%, \ell=e, \mu$. However, as pointed out earlier, to estimate the discovery potential of the LHC for lepton flavour violating signals, we will base the following analysis on the basic conservative kinematic cuts as given in Table I.

\section{B. Lepton Flavour Violation}

In this section we discuss the prospects of observing lepton flavour violating production and decay of right-handed $W_{R}$ bosons and heavy neutrinos in the left-right symmetric model described in Section $\amalg A$, due to a mixing among the right-handed neutrinos. We will focus on a mixing of right-handed neutrinos with electrons and muons, i.e. we are mostly interested in the mixing matrix elements $V_{N_{i} e}$ and $V_{N_{i} \mu}$. In turn, we will assume the case of flavour mixing with the unitarity constraints $V_{N_{i} e}^{2}+V_{N_{i} \mu}^{2}=1(i=1,2), V_{N_{1} e} V_{N_{2} e}+V_{N_{1} \mu} V_{N_{2} \mu}=0$, 

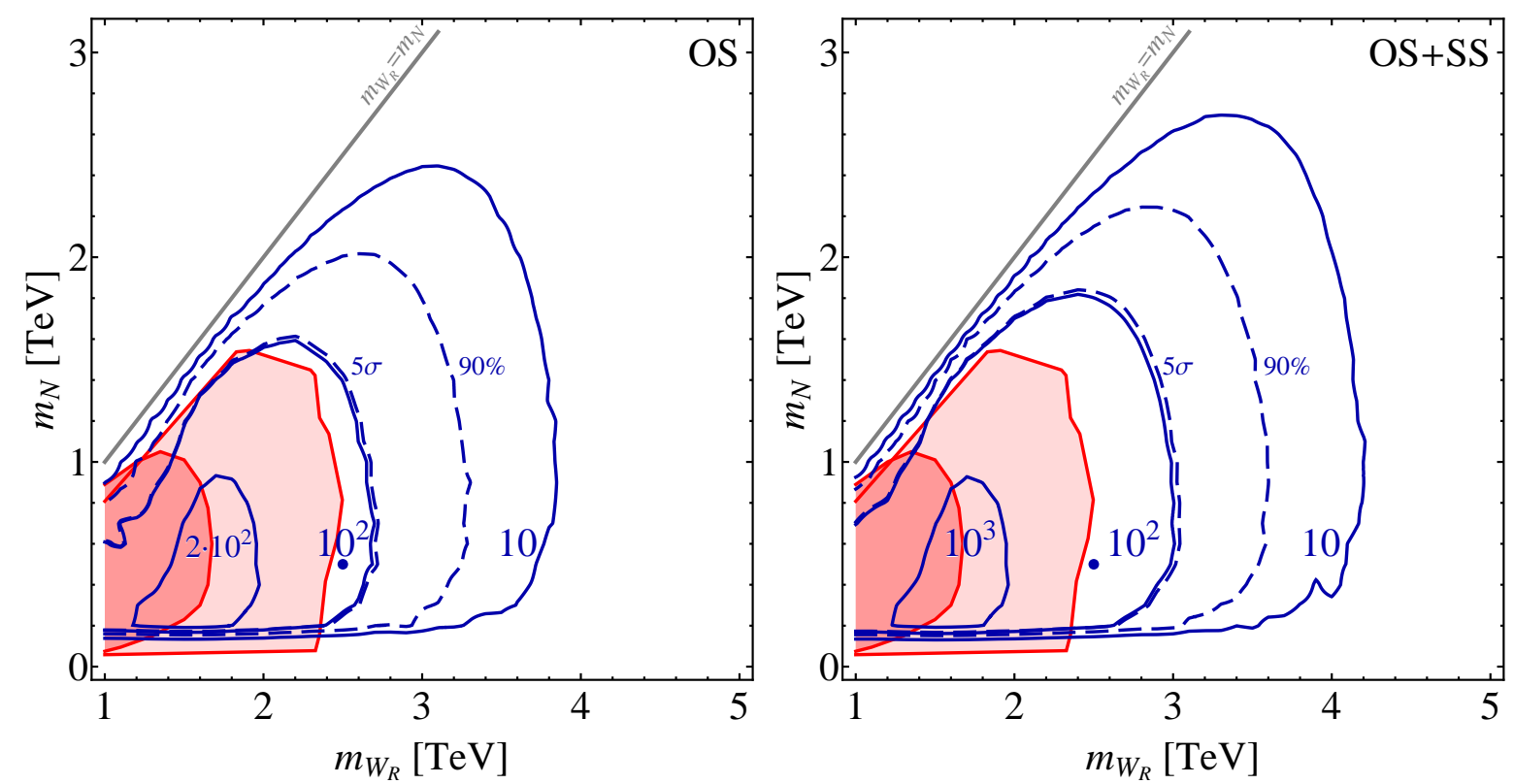

FIG. 8: The solid blue contours denote the event rates for the processes $p p \rightarrow W_{R} \rightarrow e^{ \pm} \mu^{\mp}+2$ jets (left) and $p p \rightarrow W_{R} \rightarrow e^{ \pm} \mu^{ \pm, \mp}+2$ jets (right), as a function of right-handed $W$ boson mass $m_{W_{R}}$, and the right-handed neutrino mass $m_{N}$, at the LHC with $14 \mathrm{TeV}$ and $\mathcal{L}=30 \mathrm{fb}^{-1}$. The grey contour corresponds to the kinematical threshold $m_{W_{R}}=m_{N}$. The blue dashed contours give the discovery and exclusion reach with $S / \sqrt{B}=5$ and 1.64 (90\% C.L.), respectively. The processes are calculated for maximal unitary coupling of the right-handed neutrino to $e$ and $\mu$ only with $V_{N e}=V_{N \mu}=1 / \sqrt{2}$. The red shaded areas are excluded by current LHC searches at CMS [42] (dark shaded) and ATLAS [43] (light shaded).

with either one or two heavy neutrinos light enough to be produced at the $\mathrm{LHC}^{5}$. In the first case, we will also discuss the more general scenario of a possible mixing to taus by extending the unitarity relation as $V_{N_{1} e}^{2}+V_{N_{1} \mu}^{2}+V_{N_{1} \tau}^{2}=1$. Here, we will not take into account taus in the final state $^{6}$, but a non-zero value of $V_{N_{1} \tau}$ will reduce the mixing to electrons and muons accordingly.

\section{Single Neutrino Exchange}

To simplify our discussion we first consider one right-handed neutrino in the intermediate state. That is, either only one right-handed neutrino is light enough to be produced, $m_{N_{1}}<$ $m_{W_{R}}<m_{N_{2,3}}$, or if more than one neutrino is below the threshold, the mass difference

\footnotetext{
${ }^{5}$ We always assume real mixing matrix elements, i.e. we neglect any possible $\mathrm{CP}$ violating phases in the right-handed neutrino sector.

${ }^{6}$ This is a highly interesting possibility in its own right as the reconstruction efficiencies in the leptonic channels $\tau \rightarrow \ell \bar{\nu}_{\ell} \nu_{\tau}$ with $\ell=e, \mu$ are only reduced by about a third of those for electrons and muons, simply due to the leptonic branching ratios of the $\tau$ [74]. This could make it possible to detect $\tau-\mu$ and $\tau-e$ flavour violation at the LHC.
} 
between the right-handed neutrinos is sufficiently large, $\Delta m_{N_{i} N_{j}} \gtrsim 100 \mathrm{GeV}$, such that the neutrino resonances can be individually reconstructed. In Section IVB2, we then discuss right-handed neutrinos with smaller mass differences.

The couplings of the right-handed neutrino $N_{1} \equiv N$ to electrons and muons are given by the elements $V_{N e}$ and $V_{N \mu}$ of the right-handed neutrino mixing matrix. After reconstruction and cuts, the number of opposite-sign (OS) dilepton events from the processes $p p \rightarrow W_{R} \rightarrow$ $e^{+} e^{-}\left(\mu^{+} \mu^{-}, e^{ \pm} \mu^{\mp}\right)+2$ jets, are given by

$$
\begin{aligned}
N\left(e^{+} e^{-}\right) & =\epsilon_{e e}^{\mathrm{OS}} \times \mathcal{L} \times \sigma_{e e}^{\mathrm{OS}}\left(m_{W_{R}}, m_{N}\right) \times\left|V_{N e}\right|^{4}, \\
N\left(\mu^{+} \mu^{-}\right) & =\epsilon_{\mu \mu}^{\mathrm{OS}} \times \mathcal{L} \times \sigma_{e e}^{\mathrm{OS}}\left(m_{W_{R}}, m_{N}\right) \times\left|V_{N \mu}\right|^{4}, \\
N\left(e^{ \pm} \mu^{\mp}\right) & =\epsilon_{e \mu}^{\mathrm{OS}} \times \mathcal{L} \times \sigma_{e e}^{\mathrm{OS}}\left(m_{W_{R}}, m_{N}\right) \times\left|V_{N e}\right|^{2}\left|V_{N \mu}\right|^{2},
\end{aligned}
$$

with the integrated luminosity $\mathcal{L}$, the cross section $\sigma_{e e}^{\mathrm{OS}}\left(m_{W_{R}}, m_{N}\right)$ for $e^{+} e^{-}$production with $V_{N e}=1$ (no flavour mixing), and the experimental efficiencies $\epsilon_{e e}^{\mathrm{OS}}, \epsilon_{\mu \mu}^{\mathrm{OS}}, \epsilon_{e \mu}^{\mathrm{OS}}$ due to reconstruction and cuts, for the different dilepton flavour combinations. Similar relations are obtained for same-sign dilepton pairs, with the efficiencies $\epsilon_{e e}^{\mathrm{SS}}, \epsilon_{\mu \mu}^{\mathrm{SS}}, \epsilon_{e \mu}^{\mathrm{SS}}$ and the SS cross section $\sigma_{e e}^{\mathrm{SS}}\left(m_{W_{R}}, m_{N}\right)$. The numerical values of the efficiencies are summarized in Table II. obtained in our simulation of the benchmark scenario $m_{W_{R}}=2 \mathrm{TeV}, m_{N}=0.5 \mathrm{TeV}$ with the cuts shown in Table 1 .

To determine the reach of the LHC to probe lepton flavour violation, we show in Figure 8 the event rate for the signature $p p \rightarrow W_{R} \rightarrow e \mu+2$ jets at the LHC with $14 \mathrm{TeV}$ and an integrated luminosity of $\mathcal{L}=30 \mathrm{fb}^{-1}$ for maximal mixing $V_{N e}=V_{N \mu}=1 / \sqrt{2}$. In Figure 8 (left) we show the sum of events of opposite-sign leptons, $N\left(e^{+} \mu^{-}\right)+N\left(e^{-} \mu^{+}\right)$, whereas in the right plot the charges are summed over as well, $N\left(e^{+} \mu^{-}\right)+N\left(e^{-} \mu^{+}\right)+N\left(e^{+} \mu^{+}\right)+N\left(e^{-} \mu^{-}\right)$. The blue dashed contours show the discovery reach $(S / \sqrt{B}=5$, practically coinciding with the 100 events contour) and the exclusion reach (90\% C.L. excess over background). As discussed previously, we do not optimize the cuts for each individual parameter point, which could extend the reach somewhat. Because of the simple relations in Eq. (34), the LHC reach is comparable for lepton flavour conserving and maximally violating signals, c.f. Figures 6 and 8. Effectively, the rate is halved for maximal mixing, and the shape of the regions follows from the discussion in Section IV. It should therefore be possible to probe LFV for $W_{R}$ masses up to 3-3.5 TeV and right-handed neutrino masses up to 1.5-2 TeV. For comparison, we show the CMS exclusion region with $\mathcal{L}=0.24 \mathrm{fb}^{-1}$ [42], and the ATLAS exclusion region with $\mathcal{L}=2.1 \mathrm{fb}^{-1}$ [43].

To verify the LHC sensitivity to the flavour couplings $V_{N e}$ and $V_{N \mu}$, we fix the masses $m_{W_{R}}=2.5 \mathrm{TeV}, m_{N}=0.5 \mathrm{TeV}$, and show in Figure 9 the event rates as a function of the electron coupling $V_{N e}$ for OS and OS+SS signatures, where we assume unitarity among the couplings $V_{N e}$ and $V_{N \mu}$, i.e. $V_{N e}^{2}+V_{N \mu}^{2}=1$. The dependence of the event rates on the couplings is again very simple and follows Eqs. (34). If the lepton flavour violating signature $e-\mu$ is observable, at least one of the flavour conserving signatures $e-e$ or $\mu-\mu$ can be observed as well. If $V_{N e}^{2}>1 / 2$, the neutrino couples more strongly to electrons than to muons, and from Eqs. (34) we can determine

$$
V_{N e}^{2}=\left[1+\frac{1}{2} \frac{\epsilon_{e e}^{O S}}{\epsilon_{e \mu}^{O S}} \frac{N\left(e^{+} \mu^{-}+e^{-} \mu^{+}\right)}{N\left(e^{+} e^{-}\right)}\right]^{-1},
$$

for opposite-sign signatures. The coupling parameter $\left|V_{N e}\right|$ can therefore be determined independently of the absolute normalization of the production cross section (e.g. due to 

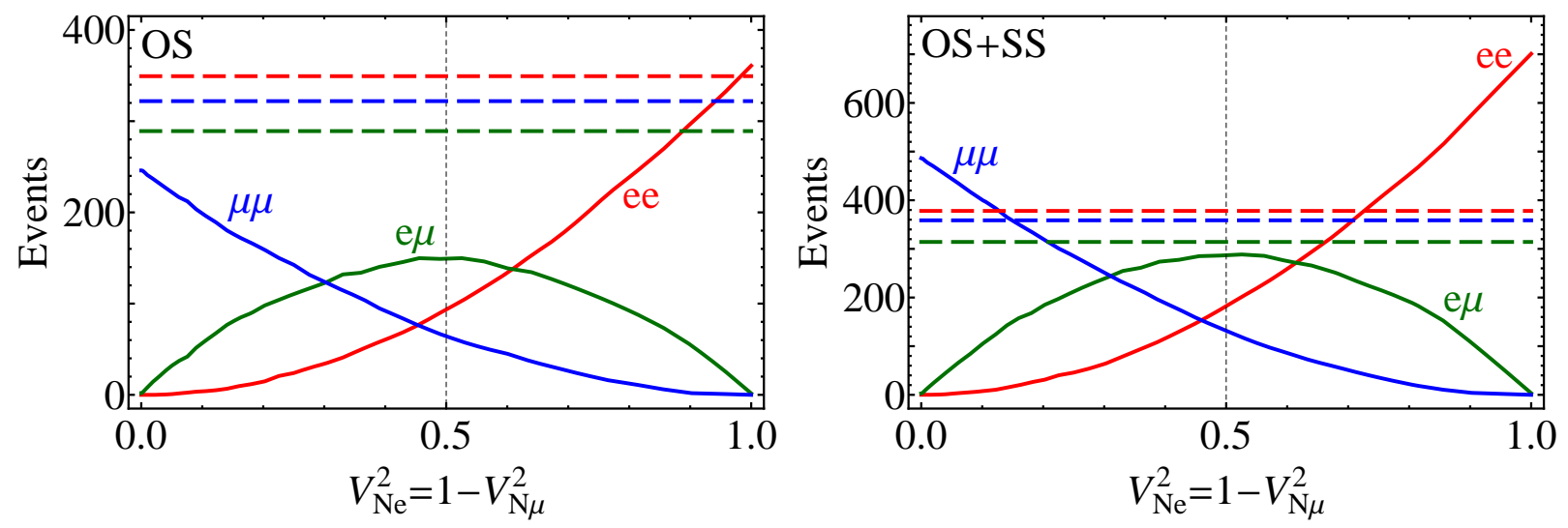

FIG. 9: Event rates for the processes $p p \rightarrow W_{R} \rightarrow e e(\mu \mu, e \mu)+2$ jets at the LHC with $14 \mathrm{TeV}$ and $\mathcal{L}=30 \mathrm{fb}^{-1}$ as a function of the right-handed neutrino-electron coupling $V_{N e}^{2}=1-V_{N \mu}^{2}$ for a right-handed $W$ boson mass $m_{W_{R}}=2.5 \mathrm{TeV}$ and right-handed neutrino mass $m_{N}=0.5 \mathrm{TeV}$. The rates are calculated using only the opposite charge sign event sample (left) and both opposite and same charge sign sample (right) of the two leptons. The horizontal dashed lines show the background to each correspondingly colored signal.

possible splitting of the gauge couplings, $g_{R} \neq g_{L}$ effects) and systematic uncertainties are reduced. If $V_{N e}^{2}<1 / 2$, we find an analogous expression, replacing $N\left(e^{+} e^{-}\right)$with $N\left(\mu^{+} \mu^{-}\right)$ in the denominator of Eq. (35). For $V_{N e}^{2} \approx 1 / 2$, Eq. (35) can be used both with $N\left(e^{+} e^{-}\right)$ and $N\left(\mu^{+} \mu^{-}\right)$, yielding two independent measurements for $V_{N e}^{2}$.

This can be generalized by dropping the unitarity condition and keeping the couplings $V_{N e}$ and $V_{N \mu}$ independent from each other. This approach is used in Figure 10, where we show the excluded region in the $V_{N e}$ and $V_{N \mu}$ parameter plane at $90 \%$ C.L., using a $\chi^{2}$ analysis of the $e e, e \mu$ and $\mu \mu$ events with both OS and OS+SS signatures, assuming no excess above the background is observed. In the given scenario with $m_{W_{R}}=2.5 \mathrm{TeV}$ and $m_{N}=0.5 \mathrm{TeV}$, couplings of the order of $V_{N e(\mu)} \approx 0.5$ can be excluded. For illustration, we also show uncertainty contours for hypothetical signals corresponding to four different choices for $\left(V_{N e}, V_{N \mu}\right)$ : (a) $(1 / \sqrt{2}, 1 / \sqrt{2})$, corresponding to maximal unitary mixing; (b) $(0.347,0.938)$, representing the unitary scenario with the minimal value of $V_{N e}$ that can be distinguished from zero; (c) $(0.937,0.348)$, representing the unitary scenario with the minimal value of $V_{N \mu}$ that can be distinguished from zero; (d) $(0.5,0.5)$, representing a nonunitary scenario close to the exclusion limit. In Figure 10, the errors on the event rates are assumed to be dominated by their statistical uncertainties.

In order to derive the sensitivity as a function of $m_{W_{R}}$ and $m_{N}$, we show in Figure 11 the minimal coupling $V_{N e}^{2}$ which can be observed at $5 \sigma$, for both OS and OS+SS leptons. The outermost contour in the plots corresponds to a signal at 90\% C.L. for maximal mixing $V_{N e}^{2}=V_{N \mu}^{2}=1 / 2$. This exclusion contour and the $5 \sigma$ discovery contour therefore correspond to the dashed contours in Figure 8, respectively. The parameter region with the largest cross section around $\left(m_{W_{R}}, m_{N}\right)=(1.6,0.3) \mathrm{TeV}$ is already excluded by LHC searches, and flavour violating right-handed neutrino-lepton couplings down to $V_{N e(\mu)}^{2} \approx 10^{-1}$ can potentially be probed at the $\mathrm{LHC}$ with $14 \mathrm{TeV}$ and $\mathcal{L}=30 \mathrm{fb}^{-1}$. 


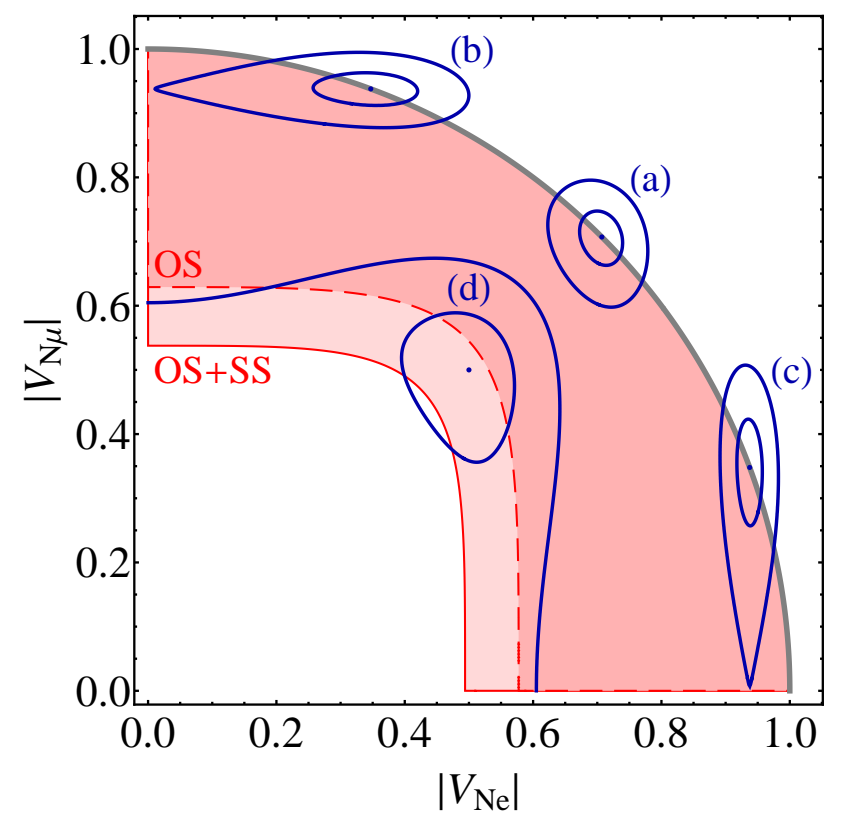

FIG. 10: Excluded regions in the $V_{N e}$ and $V_{N \mu}$ parameter plane at $90 \%$ C.L. using OS (light red shaded with solid boundary) and OS+SS (dark red with dashed boundary) event samples, assuming no excess above background. Also shown are the $1 \sigma$ and $5 \sigma$ uncertainty solid blue contours for hypothetical signals corresponding to $\left(V_{N e}, V_{N \mu}\right)=(1 / \sqrt{2}, 1 / \sqrt{2}),(0.347,0.938),(0.937,0.348)$, $(0.5,0.5)$ using OS+SS samples. The statistical analysis is based on the events $p p \rightarrow W_{R} \rightarrow$ $e e(\mu \mu, e \mu)+2$ jets at the LHC with $14 \mathrm{TeV}$ and $\mathcal{L}=30 \mathrm{fb}^{-1}$, with a $W_{R}$ boson mass of $m_{W_{R}}=$ $2.5 \mathrm{TeV}$ and right-handed neutrino mass of $m_{N}=0.5 \mathrm{TeV}$. The errors on the event rates are assumed to be dominated by their statistical uncertainty.

\section{Two Neutrino Exchange}

In general, all heavy neutrinos with $m_{N_{i}}<m_{W_{R}}$ that couple to electrons and/or muons will contribute to our dilepton signatures and have to be taken into account. When summing over the heavy right-handed neutrinos in the intermediate state, this may lead to interference effects between the different contributions. In fact in the limit of degenerate heavy neutrinos, $\Delta m_{i j}^{2} \equiv m_{N_{i}}^{2}-m_{N_{j}}^{2} \rightarrow 0$, all lepton flavour violating signals will suffer a GIM-like suppression, analogous to low energy LFV processes as described in Section IIIC. As a crucial difference to the radiative rare decays, the neutrinos are produced on-shell at the LHC with a short decay length. This leads to a decoherence of the right-handed neutrino oscillation, and the suppression is proportional to $\Delta m_{i j}^{2} /\left(m_{N} \Gamma_{N}\right)$, rather than $\Delta m_{i j}^{2} / m_{N}^{2}$. This follows from the well justified narrow width approximation for the product of the neutrino propagators $N_{i}$ and $N_{j}$ in the squared matrix element [75]

$$
\begin{gathered}
\left(p^{2}-m_{N_{i}}^{2}+i m_{N_{i}} \Gamma_{N_{i}}\right)^{-1} \times\left(p^{2}-m_{N_{j}}^{2}-i m_{N_{j}} \Gamma_{N_{j}}\right)^{-1} \approx \\
\frac{\pi C_{i j}}{2\langle m \Gamma\rangle_{i j}}\left[\delta\left(p^{2}-m_{N_{i}}^{2}\right)+\delta\left(p^{2}-m_{N_{j}}^{2}\right)\right],
\end{gathered}
$$



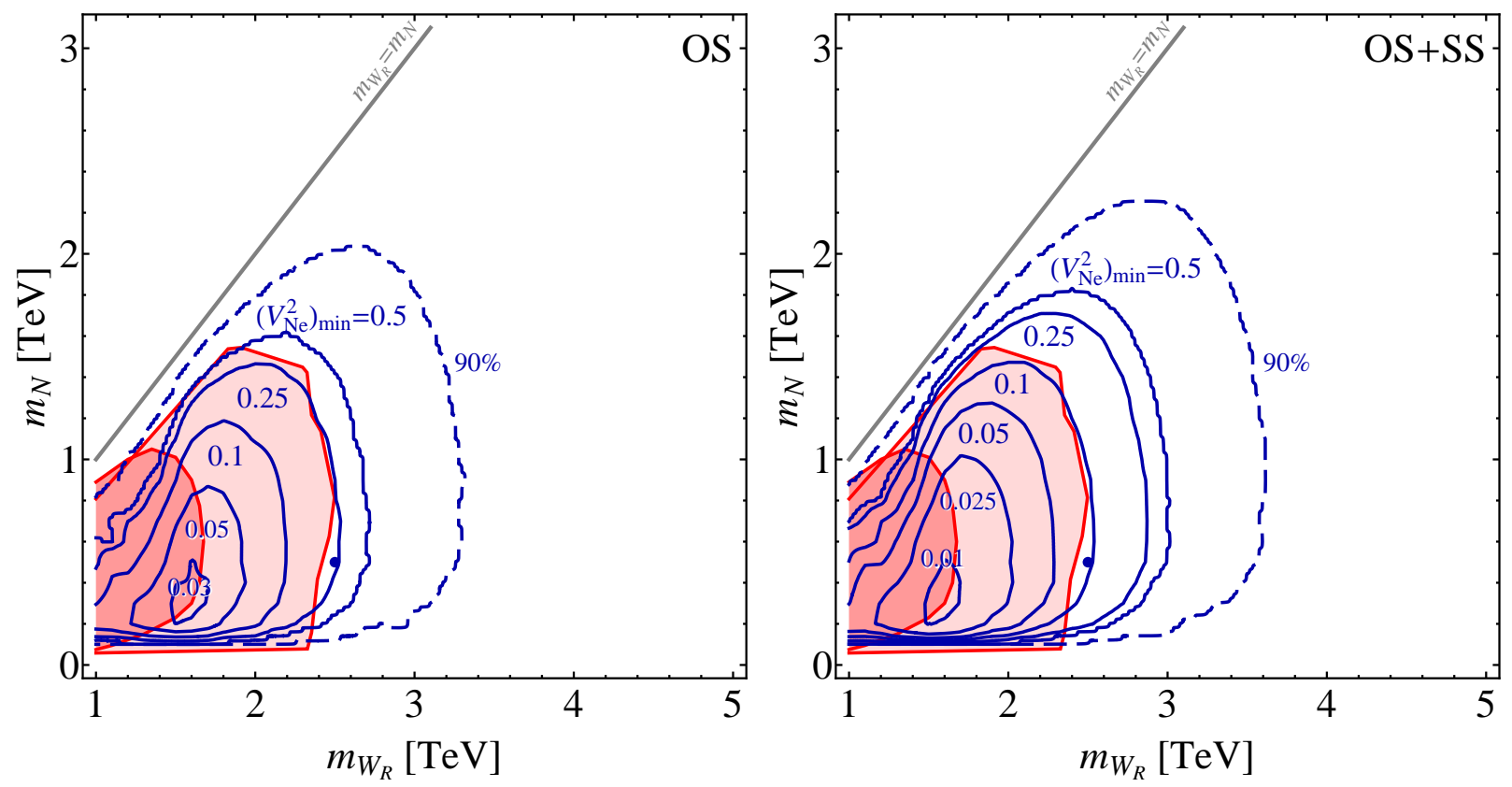

FIG. 11: Sensitivity to the coupling $V_{N e}^{2}$ as function of $m_{W_{R}}$ and $m_{N_{R}}$ at the LHC with $14 \mathrm{TeV}$ and $\mathcal{L}=30 \mathrm{fb}^{-1}$ using OS (left) and OS+SS leptons (right). The solid contours indicate a discovery sensitivity at $5 \sigma$ and the outermost contour corresponds to an exclusion at $90 \%$ C.L. for maximal mixing $V_{N e}^{2}=1 / 2$. The grey contour corresponds to the kinematical threshold $m_{W_{R}}=m_{N}$. The red shaded areas are excluded by current LHC searches at CMS [42] (dark shaded) and ATLAS [43] (light shaded).

with

$$
C_{i j}=\left(1+i \frac{\Delta m_{i j}^{2}}{2\langle m \Gamma\rangle_{i j}}\right)^{-1}, \quad \Delta m_{i j}^{2}=m_{N_{i}}^{2}-m_{N_{j}}^{2}, \quad\langle m \Gamma\rangle_{i j}=\frac{1}{2}\left(m_{N_{i}} \Gamma_{N_{i}}+m_{N_{j}} \Gamma_{N_{j}}\right)
$$

For large mass splittings, $\Delta m_{i j}^{2} \gg\langle m \Gamma\rangle_{i j}$, the factors $C_{i j}$ approach $\delta_{i j}$, i.e. the neutrino interference is suppressed and their contributions add up incoherently in the squared matrix element. For small mass splittings, $\Delta m_{i j}^{2} \ll\langle m \Gamma\rangle_{i j}$, due to Eq. (36) and the unitarity of the mixing matrix $V$ in the vertex $V_{N_{i} \ell} N_{i} \ell-W_{R}$, the flavour violating process $p p \rightarrow W_{R} \rightarrow$ $\ell_{1} \ell_{2}+2$ jets, $\ell_{1} \neq \ell_{2}$, is suppressed as $\Delta m_{i j}^{2} /\langle m \Gamma\rangle_{i j}$. When two neutrinos $N_{1,2}$ couple to $e$ and $\mu$, with a unitarity matrix described by the mixing angle $\phi$,

$$
V=\left(\begin{array}{cc}
\cos \phi & \sin \phi \\
-\sin \phi & \cos \phi
\end{array}\right)
$$

the total event rate for the process $p p \rightarrow W_{R} \rightarrow e^{ \pm} \mu^{\mp}+2$ jets is then

$$
N\left(e^{ \pm} \mu^{\mp}\right) \approx \epsilon_{e \mu}^{\mathrm{OS}} \mathcal{L}\left[\sigma\left(m_{W_{R}}, m_{N_{1}}\right)+\sigma\left(m_{W_{R}}, m_{N_{2}}\right)\right] \times \frac{1}{4} \sin ^{2}(2 \phi) \frac{\left(\Delta m_{12}^{2}\right)^{2}}{\left(\Delta m_{12}^{2}\right)^{2}+2\langle m \Gamma\rangle_{12}^{2}} .
$$

Here, $\sigma\left(m_{W_{R}}, m_{N_{i}}\right)$ is the cross section of the lepton flavour conserving process $p p \rightarrow e^{+} e^{-}+$ 2 jets with exchange of one neutrino of mass $m_{N_{i}}$ coupling only to electrons. This equation describes the event rate for all mass splitting regimes to a very good approximation: 
1. If the neutrino mass difference is larger than the experimental width of the neutrino resonances in the invariant mass distribution $m_{\ell_{2} j j}, \Delta m_{i j} \gtrsim \Gamma_{N_{i}}^{\ell j j}$, it is in principle possible to reconstruct each of the resonances $m_{N_{1,2}}$, and to determine the couplings $V_{N_{1} e}^{2}, V_{N_{1} \mu}^{2}, V_{N_{2} e}^{2}, V_{N_{2} \mu}^{2}$ of both neutrinos, as described in Section IVB 1. The individual event rate for each resonance is then

$$
N_{N_{i}}\left(e^{ \pm} \mu^{\mp}\right) \approx \epsilon_{e \mu}^{\mathrm{os}} \mathcal{L} \sigma\left(m_{W_{R}}, m_{N_{i}}\right) \times \frac{1}{4} \sin ^{2}(2 \phi)
$$

which is independent of the neutrino mass splitting.

2. If $\Gamma_{N_{i}} \ll \Delta m_{i j} \ll \Gamma_{N_{i}}^{\ell j j}$, it is not possible to resolve the individual neutrino contributions which overlap and form a single resonance at $m_{\bar{N}} \approx m_{N_{1}} \approx m_{N_{2}}$. Nevertheless, the LFV rate is still unsuppressed by the mass splitting and the total event rate in the single resonance is given by

$$
N\left(e^{ \pm} \mu^{\mp}\right) \approx \epsilon_{e \mu}^{\mathrm{OS}} \mathcal{L} \sigma\left(m_{W_{R}}, m_{\bar{N}}\right) \times \frac{1}{2} \sin ^{2}(2 \phi) .
$$

3. If $\Delta m_{i j} \ll \Gamma_{N_{i}}$, the neutrinos form a single resonance at $m_{\bar{N}}$ and the total event rate is suppressed with $\Delta m_{i j}=m_{N_{i}}-m_{N_{j}}$ as

$$
N\left(e^{ \pm} \mu^{\mp}\right) \approx \epsilon_{e \mu}^{\mathrm{OS}} \mathcal{L} \sigma\left(m_{W_{R}}, m_{\bar{N}}\right) \times \sin ^{2}(2 \phi)\left(\frac{\Delta m_{i j}}{\langle\Gamma\rangle}\right)^{2}, \quad\langle\Gamma\rangle=\left(\Gamma_{N_{i}}+\Gamma_{N_{j}}\right) / 2 .
$$

Due to the small neutrino three-body decay width, $\Gamma_{N} \propto g_{R}^{2} m_{N}^{5} / m_{W}^{4}<10^{-2} \mathrm{GeV}$ (cf. Figure (6), the regimes 1) and 2) apply for a wide range of neutrino mass splittings. In these regimes, the oscillations between right-handed neutrinos decohere. Consequently, the LFV process rates at the LHC are not suppressed and are independent of the mass splitting (unless the individual resonances can be resolved kinematically), and can be probed at the LHC for mass differences as small as $\Delta m_{N} \approx \Gamma_{N} \approx 10^{-2}-10^{-6} \mathrm{GeV}$. This is in stark contrast to low energy rare LFV processes which, as described in Section IIIC, experience a right-handed GIM-like suppression as $\Delta m_{N}^{2} / m_{W_{R}}^{2}$. For example, for two flavour $e-\mu$ mixing, the branching ratio of $\mu \rightarrow e \gamma$, Eq. (23)), can be written in our parametrization as

$$
B r(\mu \rightarrow e \gamma) \approx 2 \times 10^{-9} \sin ^{2}(2 \phi)\left(\frac{\Delta m_{12}^{2}}{m_{W_{R}}^{2}}\right)^{2}\left(\frac{2 \mathrm{TeV}}{m_{W_{R}}}\right)^{4},
$$

with similar results for the other rare processes. For the unsuppressed case with large mixing $\phi \approx \pi / 4$ and large mass difference $\Delta m_{12}^{2} \gtrsim m_{W_{R}}^{2}$, the current limits on this decay already put severe constraints on the scale $m_{W_{R}}$. As discussed in Section IIIC, the $\mu \rightarrow e$ conversion in nuclei and $\mu \rightarrow$ eee are expected to restrict the parameter space even more. On the other hand, even for modest neutrino mass splittings, e.g. $m_{N_{2}}-m_{N_{1}} \approx 50 \mathrm{GeV}$ in our benchmark scenario with $m_{N_{1}}=500 \mathrm{GeV}$ and $m_{W_{R}}=2000 \mathrm{GeV}$, the branching ratio is already suppressed by a factor $\approx 10^{-4}$ to $\operatorname{Br}(\mu \rightarrow e \gamma) \approx 4 \cdot 10^{-13}$, below the current experimental limit. The LFV event rate at the LHC would not be affected by a mass splitting of this size, and it could even be possible to reconstruct the individual neutrino mass resonances. Measurements of LFV processes at the LHC and low energy rare LFV decays therefore provide highly complementary information on the mass spectrum and the flavour couplings of the LRSM. 


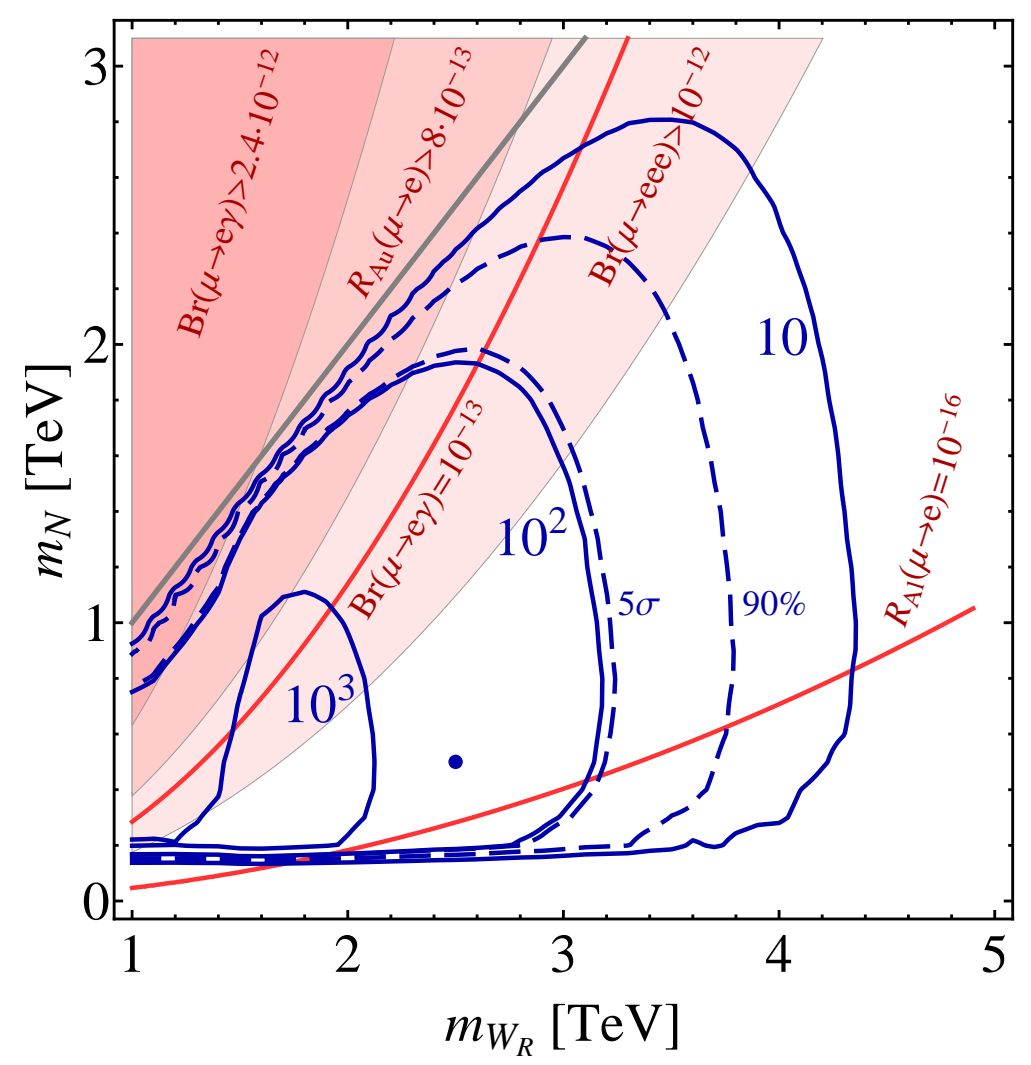

FIG. 12: Comparison of LFV event rates at the LHC and in low energy rare decays. The solid blue contours give the number of OS+SS events for the LFV signature $e^{ \pm} \mu^{ \pm, \mp}+2 j$ at the LHC with $14 \mathrm{TeV}$ and $\mathcal{L}=30 \mathrm{fb}^{-1}$. The dashed contours define the parameter region with signals at $5 \sigma$ and $90 \%$. The shaded red areas denote the parameter regions excluded by current low energy LFV limits, whereas the red contours show the expected sensitivity of planned experiments. The processes have been calculated using maximal unitary mixing between two heavy neutrinos coupling only to $e$ and $\mu$ with a $1 \%$ mass splitting, i.e. $\phi=\pi / 4$ (cf. Eq. [38), $\left(m_{N_{2}}-m_{N_{1}}\right) / m_{N_{1}}=0.01$. The spectrum of the doubly charged Higgs bosons is given by $m_{\Delta_{L, R}^{--}}=m_{W_{R}}$. The grey contour corresponds to the kinematical threshold $m_{W_{R}}=m_{N}$.

To explore this complementarity, we first compare the sensitivity of $\mu-e$ LFV processes on the masses $m_{W_{R}}$ and $m_{N}$ for maximal mixing $\phi=\pi / 4$ and a fixed $1 \%$ neutrino mass splitting, $\left(m_{N_{2}}-m_{N_{1}}\right) / m_{N}=10^{-2}$. This is shown in Figures 12 and13 for different heavy Higgs boson mass spectra: $m_{\Delta_{L, R}^{--}} / m_{W_{R}}=0.3,1,3$, mapping out the naturally expected spectral range of scales in the LRSM. As discussed in Section IIIC, the lower the Higgs boson masses are, the more constraining the processes $\mu-e$ conversion and $\mu \rightarrow$ eee become. The current limits on the rare processes already strongly constrain the parameter space, with $\mu \rightarrow e e e$ providing the most stringent bound. Even for $m_{\Delta_{L, R}^{--}} / m_{W_{R}}=3$, the parameter space with $m_{N}>m_{W_{R}}$, which is inaccessible at the LHC, is almost ruled out. For $m_{\Delta_{L, R}^{--}} / m_{W_{R}}=0.3$ the parameter space that could be probed at the LHC is almost ruled out, and only the region with rather low neutrino masses, $m_{N} \approx 200-600 \mathrm{GeV}$, is still allowed. In this mass regime, the expected COMET sensitivity on $R^{A l}(\mu \rightarrow e)=10^{-16}$ will fully probe the 

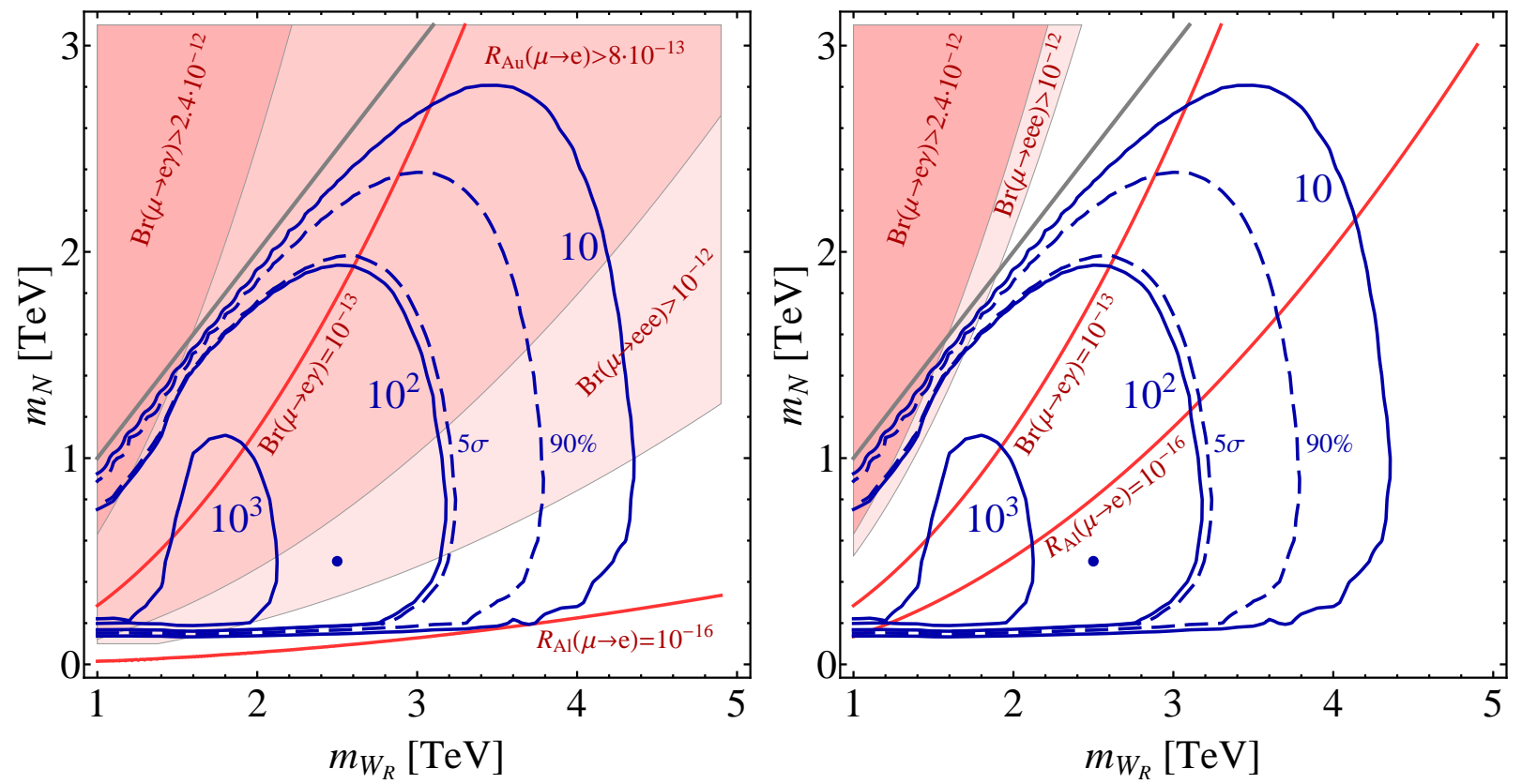

FIG. 13: As Figure 12, but with a doubly charged Higgs boson mass spectrum $m_{\Delta_{L, R}^{--}}=0.3 \times m_{W_{R}}$ (left) and $m_{\Delta_{L, R}^{--}}=3 \times m_{W_{R}}$ (right).

LHC accessible parameter space. Generically, there is a high potential that low energy LFV processes can probe the parameter space that can be also tested at the LHC, allowing for a highly detailed view of the LRSM mass spectrum and flavour mixing properties.

To explore the complementarity in probing different neutrino mass splittings and flavour mixing, we compare the sensitivity of low energy and LHC processes on $\sin ^{2}(2 \phi)$ and $\Delta m_{N} / m_{N}$ in Figure 14. Here, the LRSM mass spectrum is fixed to $m_{N}=0.5 \mathrm{TeV}$, $m_{W_{R}}=2.5 \mathrm{TeV}$ and $m_{\Delta_{L, R}^{--}}=0.66 \mathrm{TeV}$, corresponding to a light Higgs sector. As discussed above, the LFV process rate at the LHC is independent of the neutrino mass splitting until it becomes comparable and smaller than the heavy neutrino decay width at $\Gamma_{N} / m_{N} \approx 5 \cdot 10^{-8}$. It is therefore possible to probe such tiny mass differences at the LHC for mixing angles $\sin ^{2}(2 \phi) \gtrsim 10^{-1}$ in this scenario. On the other hand, the low energy processes exhibit the typical dependence $\propto \sin ^{2}(2 \phi)\left(\Delta m_{N}^{2}\right)^{2}$, and may only probe mass splittings as low as $\Delta m_{N} / m_{N} \approx 10^{-3}$.

\section{Lepton Number Violation}

So far we have only considered OS and OS+SS event signatures, but not the SS sample independently, as the significance of such a signal depends crucially on a proper treatment of the same-sign lepton background arising from charge mis-identification, mistakenly reconstructed hard leptons from jets and diboson production. Such a treatment goes beyond the scope of this work. Same-sign lepton events are of course a crucial consequence of the Majorana nature of heavy neutrinos in left-right symmetric models, and the associated lepton number violation. In the LRSM considered here, the heavy neutrinos acquire their Majorana 


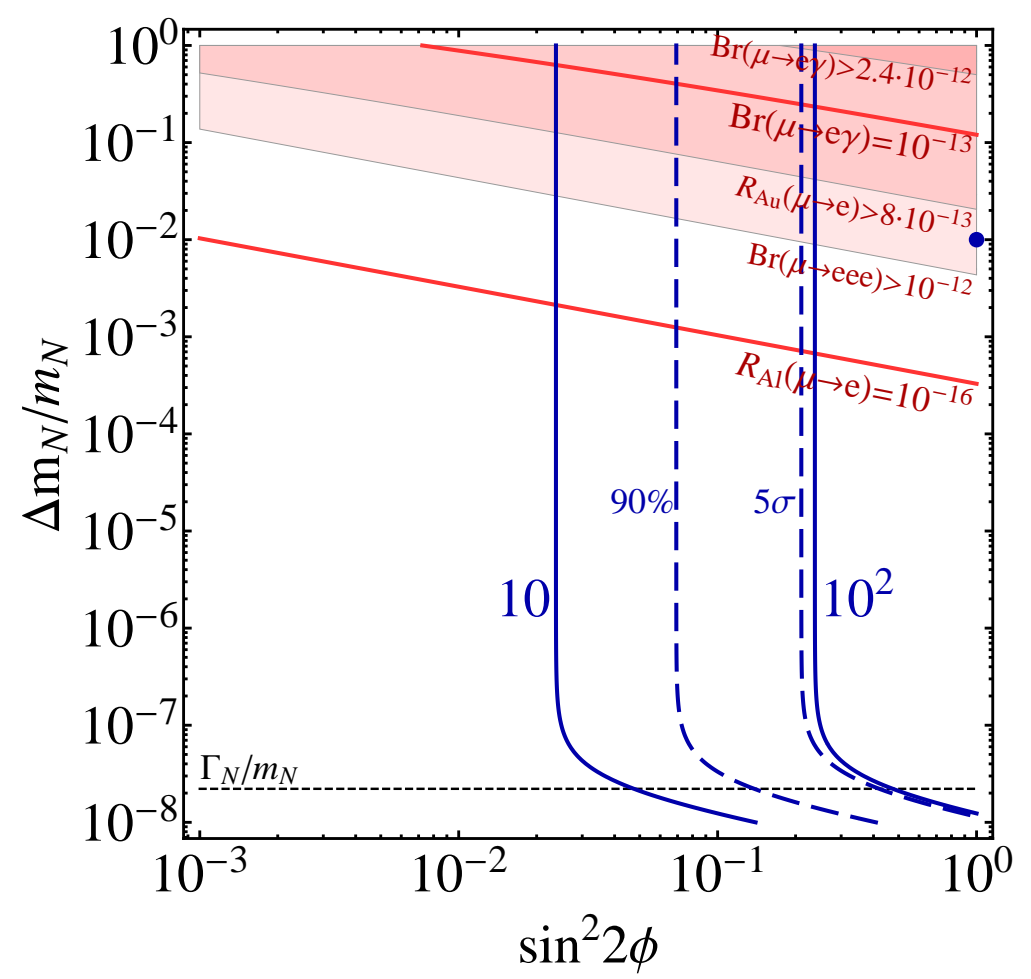

FIG. 14: As Figure 12, but showing the dependence on the heavy neutrino mixing angle parameter $\sin ^{2} 2 \phi$ and the mass splitting $\left(m_{N_{2}}-m_{N_{1}}\right) / m_{N_{1}}$. The mass scales are given by $m_{W_{R}}=2.5 \mathrm{TeV}$ and $m_{N_{1}}=0.5 \mathrm{TeV}$, with a light heavy Higgs sector, $m_{\Delta_{L, R}^{--}}=0.3 \times m_{W_{R}}$. The dashed horizontal line denotes the value for the heavy neutrino width $\Gamma_{N} / m_{N}$.

masses through the breaking of both the left-right and lepton number symmetry at the high scale $v_{R}$, much larger than the neutrino Dirac mass terms, see Eq. (8).

As a result, processes mediated by the heavy neutrinos exhibit maximal lepton number violation, with equal probabilities for the heavy neutrinos decaying into positively and negatively charged leptons. Such a scenario is technically natural to describe both light neutrino masses $\approx 0.1 \mathrm{eV}$ and heavy neutrino masses $\approx 1 \mathrm{TeV}$, if one assumes ad hoc small Dirac couplings between light and heavy neutrinos. On the other hand, accounting for the light neutrino masses within the seesaw mechanism with heavy neutrinos close to the electroweak scale would require the breaking of lepton number at a much lower scale $\mu_{\mathrm{LNV}}$, as in the inverse or linear seesaw schemes [76 80], or by invoking a proper flavour symmetry among the lepton Yukawa couplings, see e.g. [81 84]. These scenarios however lead naturally to quasi-Dirac heavy neutrinos where all lepton number violating processes are suppressed by the small mass splitting of heavy neutrinos, $\mu_{\mathrm{LNV}} / m_{N}$. From the model building viewpoint it is therefore not straightforward to predict the rate of same-sign lepton events for a given heavy neutrino mass, whereas the rate of events with opposite-sign but different flavours are generally independent of the mechanism of lepton number violation. This question will be addressed in an upcoming analysis.

Despite the above caveats, for illustration we compare the sensitivity of observing lepton number violation at the LHC with that of $0 \nu \beta \beta$ experiments in the LRSM. As pointed out in [85, 86], when staying within the LRSM, it is possible to explore the interplay between LFV 


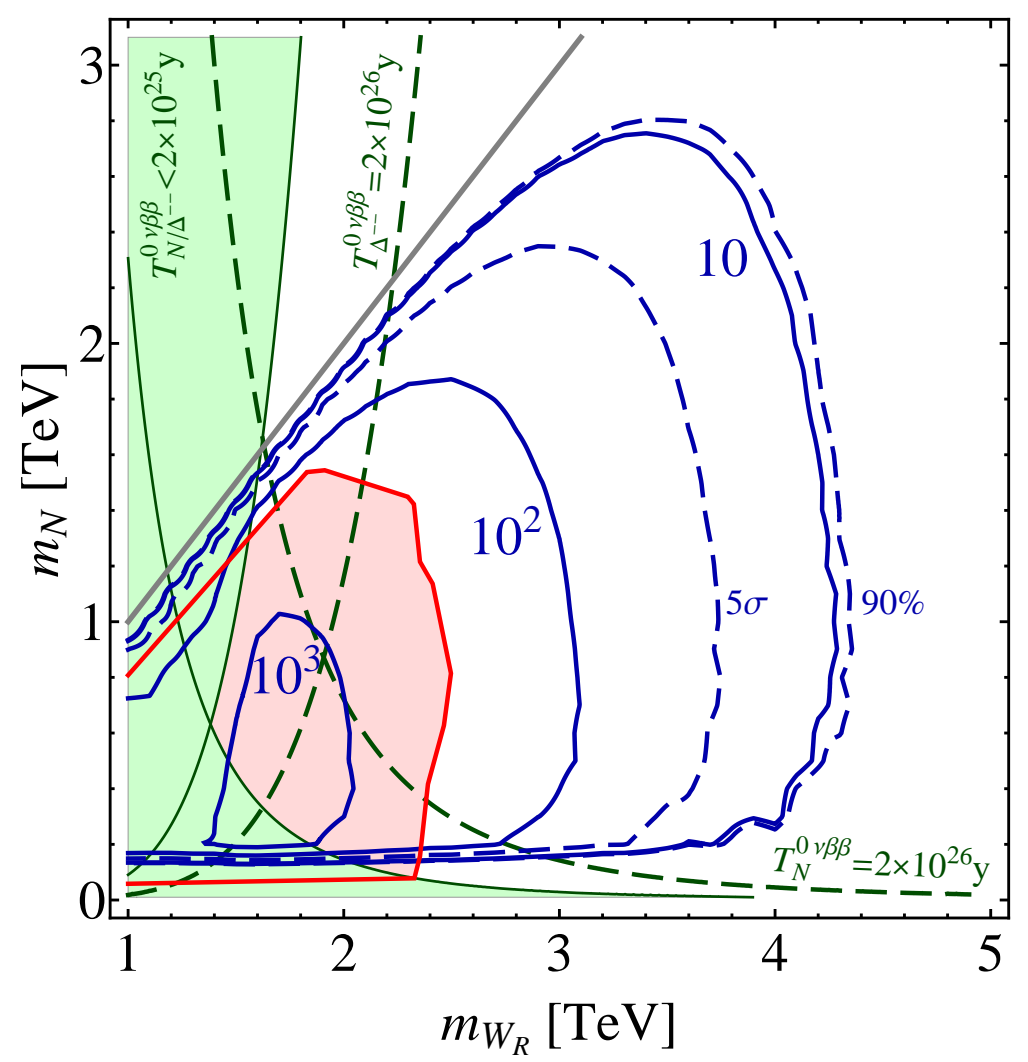

FIG. 15: Comparison of LNV event rates at the LHC and in $0 \nu \beta \beta$ experiments. The solid blue contours give the number of same-sign events for the LNV signature $e^{ \pm} e^{ \pm}+2 j$ at the LHC with $14 \mathrm{TeV}$ and $\mathcal{L}=30 \mathrm{fb}^{-1}$. The dashed blue contours define the parameter regions with a significance at $5 \sigma$ and $90 \%$, respectively, where the background is estimated using a $5 \%$ charge mis-identification probability (see discussion of Table 【I). The shaded green area denotes the parameter space excluded by $0 \nu \beta \beta$ at $T^{0 \nu \beta \beta} \approx 2 \times 10^{25}$ yrs, assuming dominant doubly-charged Higgs or heavy neutrino exchange. Correspondingly, the green dashed contours show the sensitivity of future $0 \nu \beta \beta$ experiments at $T^{0 \nu \beta \beta} \approx 2 \times 10^{26}$ yrs. The red shaded area is excluded by current LHC searches at ATLAS [43].

and LNV further. The LHC analysis in this section is the same as described in Section IV A, using identical cuts and reconstruction criteria. As illustrated in Table II, we estimate the same-sign dilepton background by assuming a $5 \%$ probability of mis-identifying the charge of a lepton of an OS event.

In Figure [15 we show the event rate of the same-sign signature $e^{ \pm} e^{ \pm}+2 j$ at the LHC with $14 \mathrm{TeV}$ and $\mathcal{L}=30 \mathrm{fb}^{-1}$. For comparison, the shaded green regions and green dashed contours represent the current limit from $T^{0 \nu \beta \beta} \gtrsim 2 \times 10^{25}$ yrs (Heidelberg-Moscow) and the future sensitivity $T^{0 \nu \beta \beta} \approx 2 \times 10^{26}$ yrs (improvement by one order of magnitude) of $0 \nu \beta \beta$ experiments. Here, we assume that neutrinoless double beta decay is either dominated by heavy neutrino exchange (Figure 2(b) and described by the effective coupling in Eq. (21)) or by Higgs triplet exchange (Figure 2(e) and described by the effective coupling in Eq. (22)). In the latter case, a light doubly charged Higgs boson mass spectrum is assumed, with $m_{\Delta_{L, R}^{--}}=0.33 \times m_{W_{R}}$. In both cases any other contribution is assumed to be zero. As 
discussed in Section ПIB, this is naturally the case for the left-right mixing contributions Figure 2(c) and (d), though the contribution from light neutrino exchange (Figure 2(a)) will always be present. Hence the sensitivities shown here would correspond to an hierarchical light neutrino mass spectrum, with a small effective mass $m_{\beta \beta}$. Due to the uncertainties described above and because of the additional dependence on the doubly charged Higgs mass and the heavy neutrino mixing matrix (here we neglect lepton flavour violation, i.e. we use $V_{N e}=1, V_{N \mu}=V_{N \tau}=0$ ), the sensitivities shown here are for illustration only. Nevertheless, it is interesting that the area currently probed by the LHC will also be tested in upcoming neutrinoless double beta decay experiments.

\section{CONCLUSIONS}

Light neutrino masses naturally arise in left-right symmetric seesaw extensions of the Standard Model, as required in order to account for current neutrino oscillations data. Lepton flavour violating effects involving charged leptons are also naturally expected in such scenarios if the masses of the heavy right-handed neutrinos present in left-right symmetric models are of the order of $1-10 \mathrm{TeV}$. Here we have considered lepton flavour violating processes induced in the production and decay of heavy right-handed neutrinos and the resulting signatures at the LHC. Either through the assumption of small Yukawa couplings or suitable model constructions implementing a low-scale seesaw, for example inverse or linear seesaw, right-handed neutrinos can have masses of order $\mathrm{TeV}$, and are hence accessible at the LHC. For this case we have derived the expected LHC sensitivities on the right-handed gauge boson and heavy neutrino masses, as well as the LFV couplings of the heavy neutrinos to charged leptons and compared the collider results with existing bounds from low energy LFV rare decays. Our discussion was mainly devoted to the first two leptonic flavours, due to their cleaner detection prospects, though extension to the tau flavour will be important, in view of good tau detection efficiencies in decays of heavy particles at the LHC [74].

Because right-handed neutrinos can be produced at gauge coupling strength in left-right symmetric models, the LHC has the potential to discover right-handed $W_{R}$ bosons up to $m_{W_{R}} \approx 3-4 \mathrm{TeV}$ and heavy neutrinos up to $m_{N_{R}} \approx 2-2.5 \mathrm{TeV}$, at $14 \mathrm{TeV}$ and $\mathcal{L}=30 \mathrm{fb}^{-1}$. No signal has been found so far, and current bounds from LHC searches are already stringent, with the excluded area extending to $\left(m_{W_{R}}, m_{N_{R}}\right) \approx(2.5,1.5) \mathrm{TeV}$. Outside these limits, LFV couplings, described by the heavy neutrino mixing matrix $V$ entering the right-handed charged current interaction $\frac{g_{R}}{\sqrt{2}} V_{N \ell} \bar{N}_{R} \gamma^{\mu} \ell_{R}$, as low as $V_{N \ell} \approx 0.3$ can still be probed at the LHC. We have also explored the complementarity of such searches with LFV probes at low energies, namely $\mu \rightarrow e \gamma, \mu \rightarrow 3 e$ and $\mu \rightarrow e$ conversion in nuclei. If the mass splitting of the heavy right-handed neutrinos is large, $\Delta m_{N}^{2} / m_{W_{R}}^{2} \gtrsim 1$, these processes already heavily constrain the presence of LFV in the right-handed neutrino sector. On the other hand, if the mass splittings are small, $\Delta m_{N}^{2} / m_{W_{R}}^{2} \lesssim 0.01$, the low energy LFV processes are GIM suppressed whereas LFV can still be observed at the LHC through the resonant production of right-handed neutrinos. This analysis provides an example of the general complementarity between LFV searches at the LHC and at low energies. Being based on high intensity experiments, low energy probes such as $\mu \rightarrow e \gamma$ have a further reach to higher scales of new physics as well as smaller LFV couplings and mixing angles. On the other hand, collider searches are limited by the available energy and luminosity, but have the potential to probe individual particles and couplings.

In this work, our focus has been on lepton flavour violating effects and the potential 


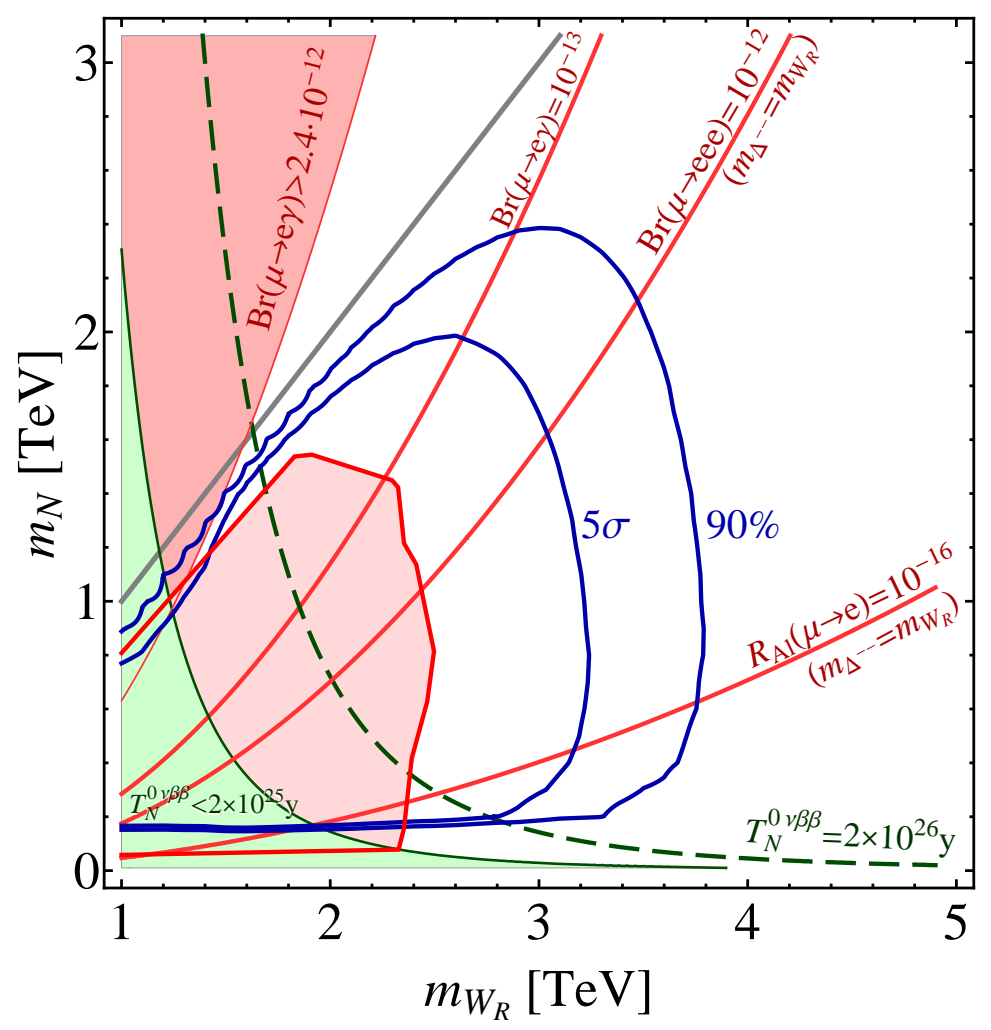

FIG. 16: Comparison of LFV and LNV event rates at the LHC and in low energy probes. The solid blue contours define the parameter region with signals of the LFV OS+SS process $p p \rightarrow W_{R} \rightarrow$ $N_{R} \ell \rightarrow e \mu+2$ jets at the LHC at $5 \sigma$ and $90 \%\left(14 \mathrm{TeV}, \mathcal{L}=30 \mathrm{fb}^{-1}\right)$. The solid gray contour corresponds to the kinematical threshold $m_{W_{R}}=m_{N}$. Overlayed are the current and expected future sensitivities of low energy LFV processes and $0 \nu \beta \beta$ (mediated by heavy neutrinos), as denoted in the plot. All processes were calculated assuming maximal flavour mixing of two heavy neutrinos $N_{1}$ and $N_{2}$ to electrons and muons with a mass difference $m_{N_{2}}-m_{N_{1}}=0.01 m_{N}$. The red shaded area in the lower left corner is excluded by current LHC searches at ATLAS.

of the LHC to probe the flavour mixing of the heavy neutrinos in left-right symmetric models. The dedicated analysis of lepton number violating effects at the LHC requires a thorough simulation of the relevant same-sign dilepton background. On the theoretical side, it also requires a detailed specification of the lepton number symmetry breaking mechanism. Within the minimal left-right symmetric model, lepton number is broken at a high scale, generating the heavy Majorana masses of the right-handed neutrinos. The resulting LNV effects at the LHC are therefore maximal, with the heavy neutrinos decaying with equal probabilities into positive and negative leptons. An overview of the sensitivities of high and low energy probes of LFV and LNV processes in this scenario is shown in Figure 16.

Especially with respect to the origin of lepton number violation, the scenario analyzed in this work is not unique, and there are theoretical consideration to address some of the issues of the minimal left-right symmetric model. Right-handed neutrinos are the messengers whose exchange yields neutrino masses through the type-I seesaw mechanism. Similarly, heavy scalar triplet exchange induces neutrino masses through the type-II seesaw. It is therefore expected that, at some level, the smallness of neutrino masses will make it difficult, 
if not preclude, to probe the physics of the heavy right-handed neutrinos. First note that having $\mathrm{TeV}$-scale right-handed gauge bosons in the minimal $\mathrm{SU}(2)_{L} \otimes \mathrm{SU}(2)_{R} \otimes \mathrm{U}(1)_{B-L}$ model discussed in Sec. IA does not, by itself, provide a fully satisfactory picture. For example, the gauge couplings in such minimal scheme have no simple $\mathrm{SO}(10)$ embedding compatible with gauge coupling unification. On the other hand right-handed neutrinos at the $\mathrm{TeV}$ scale can only be possible through the ad-hoc requirement of tiny Dirac neutrino Yukawa couplings in Eq. (6).

These shortcomings can all be naturally evaded by implementing a low scale seesaw mechanism, such as inverse [76, 80] or linear seesaw [79] within the $\mathrm{SU}(2)_{L} \otimes \mathrm{SU}(2)_{R} \otimes \mathrm{U}(1)_{B-L}$ context [77, 78]. This would not only justify the lightness of the right-handed neutrinos, without the need to invoke unnaturally small Yukawa couplings, but it also achieves a consistent gauge coupling unification within supersymmetry [87].

\section{Acknowledgements}

We thank Stephen Bieniek, Martin Hirsch and Mikael Rodriguéz for fruitful discussions. We would also like to thank Francisco del Aguila and Juan Antonio Aguilar-Saavedra for a careful reading of the manuscript and useful comments. This work was supported by the Spanish MEC under grants FPA2008-00319/FPA, FPA2011-22975 and MULTIDARK CSD2009-00064 (ConsoliderIngenio 2010 Programme), by Prometeo/2009/091 (Generalitat Valenciana), by the EU ITN UNILHC PITN-GA-2009-237920. The work of O.K. has been supported by a CPAN fellowship. S.P.D. also acknowledged financial support from DST, Government of India, SR/MF/PS-03/2009-VB-I.

[1] Super-Kamiokande collaboration, Y. Fukuda et al., Phys. Rev. Lett. 81, 1562 (1998), [hepex/9807003].

[2] SNO collaboration, Q. R. Ahmad et al., Phys. Rev. Lett. 89, 011301 (2002), [nucl-ex/0204008].

[3] KamLAND collaboration, K. Eguchi et al., Phys. Rev. Lett. 90, 021802 (2003), [hepex/0212021].

[4] M. Maltoni, T. Schwetz, M. A. Tortola and J. W. F. Valle, New J. Phys. 6, 122 (2004), hep-ph/0405172.

[5] C. Kraus et al., Eur. Phys. J. C40, 447 (2005), [hep-ex/0412056].

[6] KATRIN collaboration, A. Osipowicz et al., hep-ex/0109033.

[7] WMAP Collaboration, E. Komatsu et al., Astrophys. J. Suppl. 180, 330 (2009), [0803.0547].

[8] J. Schechter and J. W. F. Valle, Phys. Rev. D25, 2951 (1982).

[9] M. Duerr, M. Lindner and A. Merle, JHEP 1106, 091 (2011), [1105.0901].

[10] H. V. Klapdor-Kleingrothaus et al., Eur. Phys. J. A12, 147 (2001), [hep-ph/0103062].

[11] P. Minkowski, Phys. Lett. B67, 421 (1977).

[12] M. Gell-Mann, P. Ramond and R. Slansky, (1979), Print-80-0576 (CERN).

[13] T. Yanagida, (KEK lectures, 1979), ed. O. Sawada and A. Sugamoto (KEK, 1979).

[14] R. N. Mohapatra and G. Senjanovic, Phys. Rev. Lett. 44, 912 (1980).

[15] J. Schechter and J. W. F. Valle, Phys. Rev. D22, 2227 (1980).

[16] J. Schechter and J. W. F. Valle, Phys. Rev. D25, 774 (1982).

[17] G. Lazarides, Q. Shafi and C. Wetterich, Nucl. Phys. B181, 287 (1981). 
[18] M. Fukugita and T. Yanagida, Phys. Lett. B174, 45 (1986).

[19] D. Forero, S. Morisi, M. Tortola and J. W. F. Valle, JHEP 1109, 142 (2011), [1107.6009].

[20] A. Abada, C. Biggio, F. Bonnet, M. B. Gavela and T. Hambye, JHEP 12, 061 (2007), [0707.4058].

[21] F. del Aguila, J. de Blas and M. Perez-Victoria, Phys.Rev. D78, 013010 (2008), [0803.4008].

[22] F. del Aguila, J. Aguilar-Saavedra and R. Pittau, JHEP 0710, 047 (2007), [hep-ph/0703261].

[23] J. C. Pati and A. Salam, Phys. Rev. D10, 275 (1974).

[24] R. Mohapatra and J. C. Pati, Phys.Rev. D11, 2558 (1975).

[25] G. Senjanovic and R. N. Mohapatra, Phys.Rev. D12, 1502 (1975).

[26] P. Duka, J. Gluza and M. Zralek, Annals Phys. 280, 336 (2000), [hep-ph/9910279].

[27] H. Nunokawa, S. J. Parke and J. W. F. Valle, Prog. Part. Nucl. Phys. 60, 338 (2008).

[28] M. Hirsch et al., 1201.5525, Proceedings of the first workshop on Flavor Symmetries and consequences in Accelerators and Cosmology (FLASY2011).

[29] G. Barenboim and M. Raidal, Nucl.Phys. B484, 63 (1997), [hep-ph/9607281].

[30] F. del Aguila, A. Aparici, S. Bhattacharya, A. Santamaria and J. Wudka, 1111.6960.

[31] M. Hirsch, S. Morisi and J. W. F. Valle, Phys. Lett. B679, 454 (2009), [0905.3056].

[32] M. Dittmar, A. Santamaria, M. C. Gonzalez-Garcia and J. W. F. Valle, Nucl. Phys. B332, 1 (1990).

[33] P. Langacker and S. Uma Sankar, Phys.Rev. D40, 1569 (1989).

[34] Y. Zhang, H. An, X. Ji and R. N. Mohapatra, Nucl.Phys. B802, 247 (2008), [0712.4218].

[35] A. Maiezza, M. Nemevsek, F. Nesti and G. Senjanovic, Phys.Rev. D82, 055022 (2010), [1005.5160].

[36] G. Beall, M. Bander and A. Soni, Phys.Rev.Lett. 48, 848 (1982).

[37] G. Barenboim, J. Bernabeu, J. Prades and M. Raidal, Phys.Rev. D55, 4213 (1997), [hep$\mathrm{ph} / 9611347]$.

[38] J. Chakrabortty, J. Gluza, R. Sevillano and R. Szafron, 1204.0736.

[39] CDF Collaboration, T. Aaltonen et al., Phys.Rev. D83, 031102 (2011), [1012.5145].

[40] CMS Collaboration, V. Khachatryan et al., Phys.Lett. B698, 21 (2011), [1012.5945].

[41] CMS Collaboration, S. Chatrchyan et al., Phys.Lett. B701, 160 (2011), [1103.0030].

[42] CMS Collaboration, C. Collaboration, (2011), CMS-PAS-EXO-11-002.

[43] ATLAS Collaboration, G. Aad et al., 1203.5420, 9 pages plus author list (23 pages total), 4 figures, 3 tables, submitted to European Physical Journal C.

[44] LHCb Collaboration, R. Aaij et al., Phys.Rev.Lett. 108, 101601 (2012), [1110.0730].

[45] LHCb Collaboration, R. Aaij et al., 1201.5600.

[46] K. Nakamura et al., Journal of Physics G: Nuclear and Particle Physics 37, 075021 (2010).

[47] J. Polak and M. Zralek, Nucl.Phys. B363, 385 (1991).

[48] A. Pilaftsis, Phys.Rev. D52, 459 (1995), [hep-ph/9502330].

[49] M. Czakon, J. Gluza and M. Zralek, Phys.Lett. B458, 355 (1999), [hep-ph/9904216].

[50] D. V. Forero, M. Tortola and J. Valle, 1205.4018.

[51] H. V. Klapdor-Kleingrothaus, A. Dietz, H. L. Harney and I. V. Krivosheina, Mod. Phys. Lett. A16, 2409 (2001), [hep-ph/0201231].

[52] SuperNEMO Collaboration, R. Arnold et al., Eur.Phys.J. C70, 927 (2010), [1005.1241].

[53] F. Deppisch, C. Jackson, I. Nasteva and S. Soldner-Rembold, Prog.Part.Nucl.Phys. 64, 278 (2010).

[54] W. Rodejohann, Int.J.Mod.Phys. E20, 1833 (2011), [1106.1334].

[55] V. Cirigliano, A. Kurylov, M. Ramsey-Musolf and P. Vogel, Phys.Rev. D70, 075007 (2004), 
[hep-ph/0404233].

[56] MEG collaboration, J. Adam et al., Phys.Rev.Lett. 107, 171801 (2011), [1107.5547], 5 pages, 2 figures, accepted for publication at Phys. Rev. Lett.

[57] SINDRUM II Collaboration, W. H. Bertl et al., Eur.Phys.J. C47, 337 (2006).

[58] SINDRUM Collaboration, U. Bellgardt et al., Nucl.Phys. B299, 1 (1988).

[59] R. K. Kutschke, 1112.0242.

[60] COMET Collaboration, A. Kurup, Nucl.Phys.Proc.Suppl. 218, 38 (2011).

[61] W.-Y. Keung and G. Senjanovic, Phys.Rev.Lett. 50, 1427 (1983).

[62] T.-H. Ho, C.-R. Ching and Z.-J. Tao, Phys.Rev. D42, 2265 (1990).

[63] A. Ferrari et al., Phys. Rev. D62, 013001 (2000).

[64] S. N. Gninenko, M. M. Kirsanov, N. V. Krasnikov and V. A. Matveev, Phys. Atom. Nucl. 70, 441 (2007).

[65] M. Nemevsek, F. Nesti, G. Senjanovic and Y. Zhang, Phys.Rev. D83, 115014 (2011), [1103.1627].

[66] J. A. Aguilar-Saavedra, Comput. Phys. Commun. 153, 275 (2003), [hep-ph/0301101], http://www-ftae.ugr.es/protos.

[67] F. del Aguila and J. A. Aguilar-Saavedra, Nucl. Phys. B813, 22 (2009), [0808.2468].

[68] F. del Aguila, J. A. Aguilar-Saavedra and J. de Blas, 0910.2720.

[69] ATLAS Collaboration, G. Aad et al., JHEP 1110, 107 (2011), [1108.0366].

[70] M. L. Mangano, M. Moretti, F. Piccinini, R. Pittau and A. D. Polosa, JHEP 0307, 001 (2003), [hep-ph/0206293].

[71] T. Sjostrand, S. Mrenna and P. Z. Skands, JHEP 05, 026 (2006), [hep-ph/0603175].

[72] E. Richter-Was, hep-ph/0207355.

[73] P. M. Nadolsky, AIP Conf.Proc. 753, 158 (2005), [hep-ph/0412146], Contribution to the proceedings of the 15th Topical Conference on Hadron Collider Physics (HCP 2004, June 14-18, 2004, East Lansing, MI): 14 pages, 8 figures Report-no: ANL-HEP-CP-04-138.

[74] J. Aguilar-Saavedra, F. Deppisch, O. Kittel and J. Valle, Phys. Rev. D85, 091301 (2012), [1203.5998].

[75] F. Deppisch, H. Pas, A. Redelbach, R. Ruckl and Y. Shimizu, Phys.Rev. D69, 054014 (2004), [hep-ph/0310053].

[76] R. N. Mohapatra and J. W. F. Valle, Phys. Rev. D34, 1642 (1986).

[77] E. Akhmedov et al., Phys. Rev. D53, 2752 (1996), [hep-ph/9509255].

[78] E. Akhmedov et al., Phys. Lett. B368, 270 (1996), [hep-ph/9507275].

[79] M. Malinsky, J. C. Romao and J. W. F. Valle, Phys. Rev. Lett. 95, 161801 (2005).

[80] F. Bazzocchi, D. Cerdeno, C. Munoz and J. W. F. Valle, Phys.Rev. D81, 051701 (2010), [0907.1262].

[81] J. Gluza, Acta Phys.Polon. B33, 1735 (2002), [hep-ph/0201002].

[82] A. Pilaftsis and T. E. J. Underwood, Nucl. Phys. B692, 303 (2004), [hep-ph/0309342].

[83] J. Kersten and A. Y. Smirnov, Phys. Rev. D76, 073005 (2007), [0705.3221].

[84] F. F. Deppisch and A. Pilaftsis, Phys.Rev. D83, 076007 (2011), [1012.1834].

[85] V. Cirigliano, A. Kurylov, M. Ramsey-Musolf and P. Vogel, Phys.Rev.Lett. 93, 231802 (2004), [hep-ph/0406199].

[86] V. Tello, M. Nemevsek, F. Nesti, G. Senjanovic and F. Vissani, Phys.Rev.Lett. 106, 151801 (2011), [1011.3522].

[87] V. De Romeri, M. Hirsch and M. Malinsky, Phys.Rev. D84, 053012 (2011), [1107.3412]. 